\title{
Improved both sides diffusion (iBSD): A new and straightforward stabilization approach for viscoelastic fluid flows
}

\author{
C. Fernandes ${ }^{\mathrm{a}}$, M.S.B. Araujo,,b, L.L. Ferrás ${ }^{\mathrm{a}}$, J. Miguel Nóbrega ${ }^{\mathrm{a}}$ \\ a Institute for Polymers and Composites/i3N, University of Minho, Campus de Azurém, 4800-058 Guimarães, Portugal

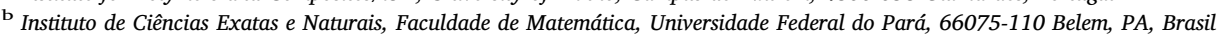

\section{A R T I C L E I N F O}

\section{Keywords:}

OpenFOAM

Upper-convected Maxwell model

Sudden contraction flow

Flow around a cylinder

\begin{abstract}
A B S T R A C T
This paper reports the developments made to improve the numerical stability of the open-source finite-volume computational library OpenFOAM ${ }^{\star}$ developed for the numerical computation of viscoelastic fluid flows described by differential constitutive models. The improvements are based on the modification of the both-sides diffusion technique, named improved both-sides diffusion (iBSD), which promotes the coupling between velocity and stress fields. Calculations for two benchmark 2D case studies of an upper-convected Maxwell (UCM) fluid are presented and compared with literature results, namely the 4:1 planar contraction flow and the flow around a confined cylinder. The results obtained for the first case are computed in five meshes with different refinement levels and are compared with literature results. In this case study it was possible to achieve steady-state converged solutions in the range of Deborah numbers tested, $D e=\{0,1,2,3,4,5\}$, for all meshes. The corner vortex size predictions agree well with the literature and a relative error below $0.6 \%$ is obtained for $D e \leq 5$. In the flow around a confined cylinder, steady-state converged solutions were obtained in the range of Deborah numbers tested, $D e=\{0,0.3,0.6,0.8\}$, in four consecutively refined meshes. The predictions of the drag coefficient on the cylinder are similar to reference data with a relative error below $0.08 \%$. For both test cases the developed numerical method was shown to have a convergence order between 1 and 2 , in general very close to the latter. Moreover, the results presented for both case studies clearly extend the previous ones available in the literature in terms of accuracy. This was a direct consequence of the capability of performing the calculation with more refined meshes, than the ones employed before.
\end{abstract}

\section{Introduction}

The use of computational fluid dynamics (CFD) increased significantly over the last decades, mainly due to the development of better and faster computers that allow the use of more realistic models, and due to the development of more accurate and efficient numerical methods. One of the approaches widely used in CFD is the finite-volume method (FVM).

Since the latest 1960s various FVM codes were developed to simulate Newtonian fluid flows (e.g. [1-3]). In the 1990s a significant interest in the use of FVM to simulate flows of viscoelastic fluids was evident, mainly due to their inherent economy of computational resources. The majority of the codes developed were initially limited to orthogonal staggered grids, as in the works of Yoo and Na [4], Sasmal [5] or Xue et al. [6], amongst others. Afterwards, other FVM codes have been developed to predict viscoelastic flows in more complex geometries. For example, Huang et al. [7] developed a method able to cope with unstructured meshes to simulate inertialess flow of Phan-Thien-
Tanner (PTT) fluids in eccentric bearings. Oliveira et al. [8] developed a collocated FVM based on non-orthogonal block-structured grids for UCM and Oldroyd-B viscoelastic fluids which was subsequently extended to handle PTT fluids [9]. The method was applied to predict the slip-stick flow, the flow around a confined cylinder and flow in planar contractions [10,11]. Favero et al. [12] implemented several viscoelastic differential models in the open-source software package Open$F O A M^{\circ}$ using the discrete elastic-viscous stress splitting formulation. OpenFOAM ${ }^{\circ}$ uses a collocated FVM, storing all variables at cell centers and is capable of handling complex mesh types such as tetrahedral and polyhedral meshes. The major drawback of the numerical algorithm implemented by Favero et al. [12] to deal with viscoelastic fluid flows in OpenFOAM ${ }^{\circ}$ is that it is prone to numerical instabilities caused by a velocity-stress decoupling, which are worst when there is no contribution from the solvent viscosity, as happens for the UCM model. More recently, Habla et al. [13] presented a new formulation for the discretization of the divergence of the viscoelastic stress tensor for the collocated FVM implemented in OpenFOAM ${ }^{\circ}$. The reformulation

\footnotetext{
* Corresponding author.

E-mail address: silvino@ufpa.br (M.S.B. Araujo).
} 
allowed for a semi-implicit handling of the constitutive equation, which promotes the numerical stability of the code developed. The validation was done using planar and square-square contraction flows of a simplified Phan-Thien-Tanner (SPTT) fluid.

The rheological behavior of non-Newtonian viscoelastic fluids is complex, requiring the use of non-linear constitutive equations to obtain realistic predictions of the fluid flow. However, the use of simple constitutive equations, such as the UCM and Oldroyd-B models [14] or the White-Metzner [15] model, is very challenging from the numerical point of view. Some characteristic difficulties found when using these models are associated with stress singular behavior near sharp corners or in stagnation points. Hence, these constitutive models are very relevant to test the accuracy and robustness of new numerical methods for viscoelastic fluids. Indeed, the most severe numerical difficulties are commonly attributed to the UCM equations $[7,16]$, when compared with other constitutive differential models.

Two popular benchmark case studies usually employed to test the UCM fluid flow in newly developed viscoelastic codes, are the 4:1 planar sudden contraction flow and the flow around a confined cylinder [17]. The former case is a simple geometry that can be discretized with orthogonal grids, but generates locally complex flows, due to the high stress gradients in the vicinity of the re-entrant corner. For the flow past a confined cylinder, the maximum Deborah number that promotes a steady solution can be limited either by the development of thin stress layers on the cylinder surface and along the centerline in the cylinder wake or because the flow is prone to viscoelastic (physical) instabilities.

Another important issue in the simulation of viscoelastic flows is the accuracy of the numerical predictions. It is known that the first-order upwind scheme (UDS) used in the discretization of the advective terms is the most stable method, but generates inaccuracies due to excessive artificial diffusion. The application of bounded high-resolution schemes (HRS) to discretize the advective terms in the constitutive equation improves the accuracy of the computations, and were employed by Alves et al. [10] in the simulation of the 4:1 planar sudden contraction flow, and by Alves et al. [11] for the flow around a confined cylinder.

In this work we propose a methodology to improve the stability of the viscoelasticFluidFoam solver available in OpenFOAM ${ }^{\circ}$, aiming to increase the numerical stability and accuracy when dealing with complex fluid flows. The improvements were based on a modified version of the both-sides diffusion (BSD) technique proposed by Guénette and Fortin [18], and are simpler to apply than other methodologies proposed so far for the same purpose $[13,16,19]$. Basically, in the proposed stabilization approach the explicit contribution of the BSD technique is discretized using an extended computational stencil, which is done just by changing the usual Laplacian operator in the traditional BSD technique by the divergence of the velocity gradient field. Due to its relation with the classic BSD, this methodology was designated improved both-sides diffusion (iBSD). In addition, we used a deferred correction approach for the discretization of the advective terms, as described in Pimenta and Alves [16], which allowed to further enhance the accuracy and stability of the results obtained. To verify the developed code, the results are compared with available results for the benchmark problems of viscoelastic flow in a 4:1 planar sudden contraction and flow around a confined cylinder.

The viscoelastic solver with the proposed enhancements in terms of stability is integrated in the version 4 of foam-extend, publicly available for download at https://sourceforge.net/p/foam-extend/foam-extend$4.0 / \mathrm{ci} / \mathrm{master} /$ tree/ReleaseNotes.txt. To facilitate its use a tutorial is also provided in https://sourceforge.net/p/foam-extend/foam-extend4.0/ci/master/tree/tutorials/viscoelastic/viscoelasticFluidFoam/UCM.

The remainder sections of this paper are organized as follows: Section 2 presents the governing equations to be solved and the improvements made to stabilize the numerical procedure. In Section 3, the results obtained with the newly developed numerical code for the two benchmark case studies are presented, discussed and compared with results from the literature. The paper ends with the main conclusions.

\section{Governing equations and numerical method}

This section presents the mathematical formulation and the methodology adopted to enhance the numerical stability of the developed code.

\subsection{Governing equations}

The basic equations to be solved are those for incompressible and isothermal laminar flow of an UCM fluid, namely the continuity equation,

$\nabla \cdot \mathbf{u}=0$

and the momentum equation,

$\frac{\partial(\rho \mathbf{u})}{\partial t}+\nabla \cdot(\rho \mathbf{u u})=-\nabla p+\nabla \cdot \tau_{P}$

together with a constitutive equation for the polymeric extra stress tensor $\tau_{P}$, which describes the relation between the stress and the fluid deformation history. In the previous equations, $\mathbf{u}$ is the velocity vector, $\rho$ the fluid density, $t$ the time and $p$ the pressure.

For the extra-stress tensor $\tau_{P}$, the upper-convected Maxwell differential equation is used:

$\tau_{P}+\lambda \tau_{P}^{\nabla}=\eta_{P}\left[\nabla \mathbf{u}+(\nabla \mathbf{u})^{T}\right]$

where $\lambda$ is the relaxation time, $\eta_{P}$ the polymer viscosity and $\stackrel{\nabla}{\tau}_{P}$ denotes the upper-convected time derivative, defined as:

$\tau_{P}^{\nabla}=\frac{\partial \tau_{P}}{\partial t}+\nabla \cdot\left(\mathbf{u} \tau_{P}\right)-(\nabla \mathbf{u})^{T} \cdot \tau_{P}-\tau_{P} \cdot \nabla \mathbf{u}$

The case studies which will be addressed in this work are all for steady flows, but the numerical method used approaches the steadystate by a time marching sequence. Hence, the time derivatives in Eqs. (2) and (4) are retained. The UCM model simplifies to the Newtonian fluid model when $\lambda=0$.

\subsection{The improved both-sides diffusion (iBSD) method}

The equations presented in Section 2.1 are discretized using the finite-volume method implemented in the OpenFOAM ${ }^{\circ}$ framework. Different implementations in OpenFOAM ${ }^{\circ}$ have been done recently $[12,13,16,20-22]$, but for the UCM model, to the best of our knowledge, there are no published studies using OpenFOAM ${ }^{\circ}$. To improve the numerical stability of the viscoelastic code, a different procedure is proposed in this work, which is a modification to the one already present in the OpenFOAM ${ }^{\circ}$ framework [12]. This new procedure promotes the coupling between stress and velocity fields.

Following the traditional both-sides diffusion (BSD) approach [12], in order to increase the numerical stability, an additional diffusive term is introduced in both sides of the momentum equation, Eq. (2), to obtain:

$\frac{\partial(\rho \mathbf{u})}{\partial t}+\nabla \cdot(\rho \mathbf{u u})-\nabla^{2}\left(\eta^{\text {ไर}} \mathbf{u}\right)=-\nabla p+\nabla \cdot \tau_{P}-\nabla^{2}\left(\eta^{\grave{\lambda}} \mathbf{u}\right)$

in which $\nabla^{2}$ is the Laplacian operator, $\eta^{\star}$ is a positive parameter, the terms on the left hand side are discretized implicitly (incorporated into the coefficients of the algebraic equations) and those on the right hand side are discretized explicitly (incorporated into the source term of the algebraic equations). The major drawback of the BSD method, which was implemented in the first version of the differential viscoelastic solvers implemented in OpenFOAM ${ }^{\circledR}$ [12], is that it is prone to produce velocity and stress checkerboard fields for null solvent viscosity, as happens for the UCM model. This occurs because the coupling between the velocity and stress fields is not assured [13], as will be shown below with an example. 
To promote the velocity-stress coupling we were inspired on the methodology presented in Guénette and Fortin [18] for the FEM. The main purpose was to enlarge the computational cell used to discretize the explicit (rhs of Eq. (5)) diffusive term. Hence, instead of the Laplacian operator of the velocity field, used in the traditional BSD method, we compute the divergent of the velocity gradient [23]. In this way the momentum conservation equation Eq. (5) is given by:

$\frac{\partial(\rho \mathbf{u})}{\partial t}+\nabla \cdot(\rho \mathbf{u u})-\nabla^{2}\left(\eta^{\text {th }} \mathbf{u}\right)=-\nabla p+\nabla \cdot \tau_{P}-\nabla \cdot\left(\nabla\left(\eta^{\text {th}} \mathbf{u}\right)\right)$

The proposed formulation, named improved both-sides diffusion (iBSD), proved to be more stable than the BSD, due to the different numerical discretization of $\nabla^{2}\left(\eta^{\star} \mathbf{u}\right)$ and of $\nabla \cdot\left(\nabla\left(\eta^{\star} \mathbf{u}\right)\right)$, respectively on the left and right hand sides of Eq. (6), as it will be shown hereafter.

Using Gauss theorem, the numerical discretization of the Laplacian operator, $\nabla^{2}\left(\eta^{\star} \mathbf{u}\right)$, is given by:

$$
\begin{aligned}
& \int_{V_{P}} \nabla^{2}\left(\eta^{\text {ॠ }} \mathbf{u}\right) d V=\quad \sum_{f} \mathbf{S}_{f} \cdot\left(\eta^{\text {ॠ }} \nabla \mathbf{u}\right)_{f} \\
& =\quad \sum_{f} \eta_{f}^{\text {訬 }} \mathbf{S}_{f} \cdot(\nabla \mathbf{u})_{f} \\
& =\sum_{f} \eta_{f}^{\sharp}\left\|\mathbf{s}_{f}\right\| \frac{\mathbf{u}_{N}-\mathbf{u}_{P}}{\|\mathbf{d}\|}
\end{aligned}
$$

where $\mathbf{S}_{f}$ is the normal vector to face $f, \mathbf{u}_{N}$ and $\mathbf{u}_{P}$ are the velocity in the cell centers which share face $f$ and $\mathbf{d}$ is the distance vector between those cells centers (see Fig. 1). Using Eq. (7), the face gradient of $\mathbf{u}$ is calculated from the two known values around the face.

On the other hand, the cell-centered gradient for the two cells sharing the face can be computed as:

$(\nabla \mathbf{u})_{P}=\frac{1}{V_{P}} \sum_{f} \mathbf{S}_{f} \mathbf{u}_{f}$

which can be linearly interpolated to the face:

$(\nabla \mathbf{u})_{f}=w(\nabla \mathbf{u})_{P}+(1-w)(\nabla \mathbf{u})_{N}$

where $w$ is the weight factor.

Using Eqs. (8) and (9), the numerical discretization of the $\nabla \cdot\left(\nabla\left(\eta^{\star} \mathbf{u}\right)\right)$ operator is given by:

$$
\begin{aligned}
& \int_{V_{P}} \nabla \cdot \quad=\quad \sum_{f} \mathbf{S}_{f} \cdot\left(\eta^{\text {मे }} \nabla \mathbf{u}\right)_{f} \\
& =\quad \sum_{f} \eta_{f}^{\sharp} \mathbf{S}_{f} \cdot(\nabla \mathbf{u})_{f} \\
& =\quad \sum_{f} \eta_{f}^{\not} \mathbf{S}_{f} \cdot\left[w(\nabla \mathbf{u})_{P}\right. \\
& \left.+(1-w)(\nabla \mathbf{u})_{N}\right] \\
& =\sum_{f} \eta_{f}^{\text {岃}} \mathbf{S}_{f} \cdot\left[w\left(\frac{1}{V_{P}} \sum_{f \in P} \mathbf{S}_{f} \mathbf{u}_{f}\right)\right. \\
& \left.+(1-w)\left(\frac{1}{V_{N}} \sum_{f \in N} \mathbf{S}_{f} \mathbf{u}_{f}\right)\right]
\end{aligned}
$$

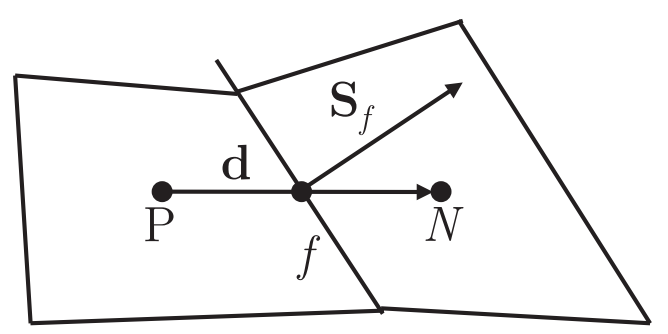

Fig. 1. Vectors $\mathbf{d}$ and $\mathbf{S}_{f}$ of diffusion term discretization. where $V_{P}$ and $V_{N}$ are the cells volume with centroids $P$ and $N$, respectively. It should be noticed that both face velocities $\mathbf{u}_{f}$ present in Eq. (10) are obtained using Eq. (8), thus depend on $P$ and $N$ neighbor cells.

Although both of the above-described discretizations are secondorder accurate, Eq. (10) uses a larger computational stencil than Eq. (7), which promotes the coupling between velocity and stress fields, and thus the oscillations are removed. It can be shown that the coupling comes from an extra term (added to the momentum equation when performing this type of discretization) representative of a central difference approximation to the fourth order velocity derivative (analogue to what is presented for pressure-velocity coupling in pages $196-200$ of the book of Ferziger and Perić [3]).

To illustrate the advantages obtained from the proposed approach, Fig. 2 shows the velocity and stress profiles for the Poiseuille flow of an UCM fluid, obtained with the original formulation and with the modified approach proposed in this work. The geometry is a rectangular channel with ratio $L / H=10$, where $L$ and $H$ are the length and height of the channel, respectively. A uniform mesh with 250 and 28 cells along the length and height, respectively, was used, which results in a $\Delta x / L$ and $\Delta y / H$ of 0.004 and 0.036 , respectively. The Reynolds and Deborah numbers for this study were $R e=\rho U H / \eta_{P}=0.001$ and $D e=\lambda U / H=0.03$, where $U$ is the inlet mean velocity. The following boundary conditions were considered: uniform velocity profile and null extra stress tensor at the inlet, no-slip at the walls and null pressure at the outlet. Both problems converged to the same residual criterion of $10^{-6}$ using a normalized time-step of $\Delta t /(L / U)=10^{-4}$. As can be seen in Fig. 2, the velocity and stress profiles obtained by the original velocitystress coupling formulation shows a checkerboard pattern, which disappears when the proposed formulation is used.

\subsection{Solution procedure}

The basis for the implementation of this new approach was the viscoelasticFluidFoam solver, developed by Favero et al. [12], in the OpenFOAM ${ }^{\circ}$ package. Pressure-velocity coupling was accomplished using segregated methods, in which the continuity equation is used to formulate an equation for the pressure, using a semi-discretized form of Eq. (1) [3]. The resulting equation set is solved by a segregated approach, using iterative algorithms with under-relaxation, such as the SIMPLE method [24]. The time derivatives in the momentum and constitutive equations are discretized with the first-order implicit Euler scheme. As stated before, the time marching is used only for relaxation purposes as we will just be looking for the steady-state solution of the case studies presented below. Thus, the method used to discretize the transient term does not affect the accuracy of the steady-state result. The advective terms in the momentum and constitutive equations are discretized using high-resolution schemes. In this work we used the same discretization schemes for the advective terms as Alves et al. [10] and [11] (MINMOD scheme of Harten [25] and SMART scheme of Gaskell and Lau [26]), allowing the comparison between our and their results. However, notice that other schemes could have been used in this study, as for example the CUBISTA scheme [27], which is widely used for viscoelastic fluid flows due to his improved iterative convergence properties. As discussed in detail in Pimenta and Alves [16], for viscoelastic fluid flow, the implementation of HRS for the extrastress components is more stable using a component-wise deferred correction approach, which was also followed in this work. We recall that in OpenFOAM ${ }^{\circ}$ the advective terms are first linearized using an explicit construction of the flux (see page 144 of Jasak [28]). The diffusive term in the momentum balance is discretized using second-order accurate linear interpolation. A second-order Gaussian discretization is applied for source terms. The velocity gradient is calculated using a fourth-order accurate least-squares approach, where at first the standard least-square gradient [29] is assembled and then, the fourth-order correction is added to the second-order accurate gradient to complete the accuracy. 


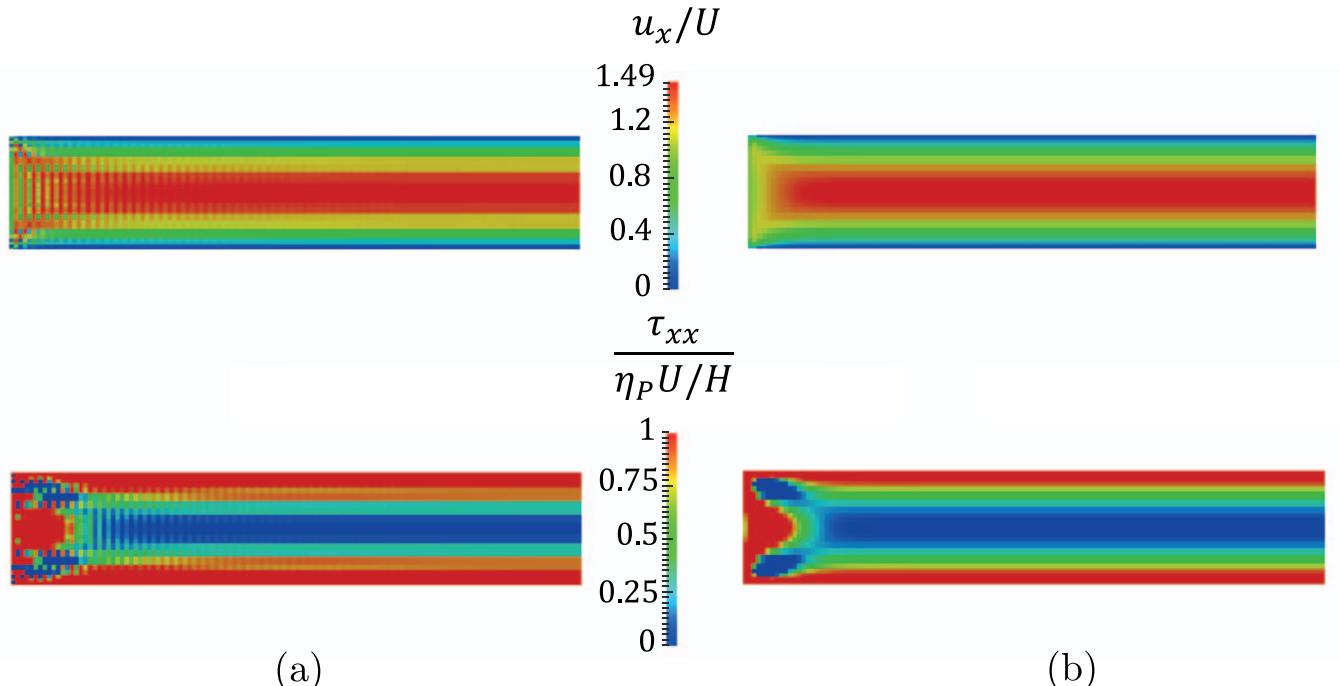

(a)

(b)

Fig. 2. Predicted velocity and stress profiles for the Poiseuille flow of an UCM fluid with the (a) original (BSD) and (b) proposed (iBSD) approaches.

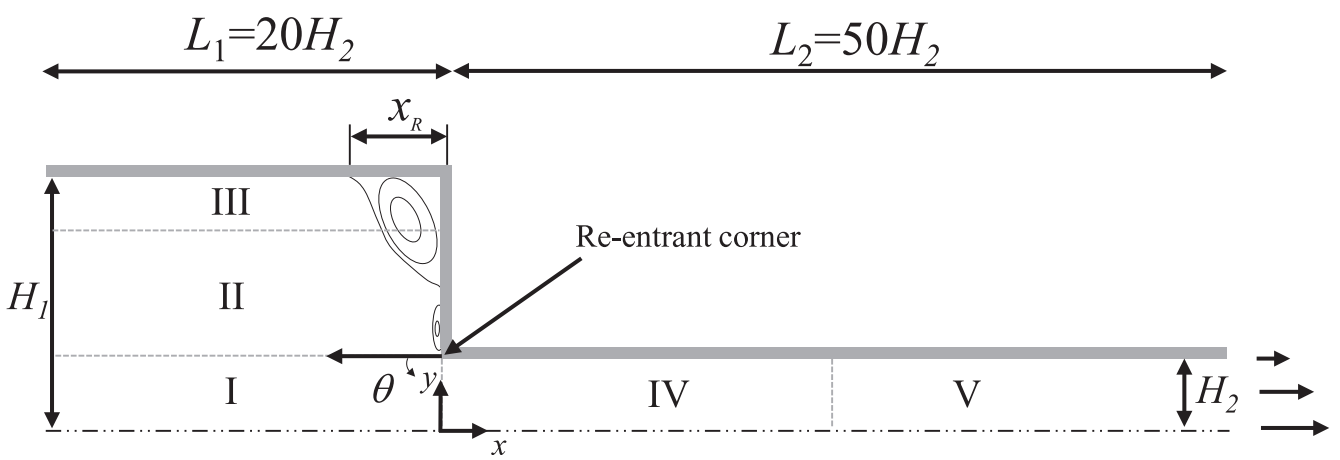

The algorithm used to solve the governing equations can be summarized in the following steps:

1. Solve the momentum balance Eq. (6), for a given initial field of velocity $\mathbf{u}$, pressure $p$, and extra-stress $\tau_{P}$, to obtain a velocity field $\mathbf{u}^{\star}$ that does not necessarily comply with the continuity equation Eq. (1).

2. Based on the SIMPLE algorithm [24], using the tentative velocity field $\mathbf{u}^{\star}$, obtained in 1 ., the new pressure correction field $p^{\star}$ is estimated solving a Poisson-type equation for pressure, that is devised through the continuity equation, Eq. (1). Subsequently, the correction of velocity and pressure fields is carried out, leading to a new velocity $\mathbf{u}^{\star \star}$, which satisfies the continuity and momentum equations. In this step the SIMPLE algorithm was used to obtain $p^{\star}$ and $\mathbf{u}^{\star \star}$; more details of the SIMPLE procedure implemented in OpenFOAM ${ }^{\circ}$ can be found in [12].

3 . Using the corrected velocity field $\mathbf{u}^{\star \star}$, solve the constitutive equation Eq. (3) to estimate the new extra-stress tensor field $\tau_{P}$.

4. Repeat steps 1-3 for a given number, $n$, of steps ( $n=1$ in this work, to obtain steady-state solutions) to generate more accurate solutions in transient flows and reducing the explicitness of the method. Only the previous time-step values of $\mathbf{u}$ and $\tau_{P}$ are not updated in these inner iterations.

5. Advance the time, update the old $\mathbf{u}$ and $\tau_{P}$ fields, and repeat again steps 1-4 until convergence is achieved.

Notice that after each discretized governing equation is solved, under-relaxation is applied to the solution (see page 115 of Jasak [28]). The relaxation factors employed were 0.3 for the pressure and stress fields and 0.5 for the velocity field. The Poisson-type equation for
Fig. 3. Schematic representation of the $4: 1$ planar sudden contraction. pressure is solved with a conjugate gradient method with Cholesky preconditioner and the velocity and stress linear systems are solved using BiCGstab with an Incomplete Lower-Upper (ILU) preconditioning [30-32]. The absolute tolerance for pressure, velocity and stress fields was set as $10^{-20}$. The simulations were stopped when the solution functionals (corner vortex size or drag coefficient) become invariant in the third decimal place.

\section{Case studies}

Sections 3.1 and 3.2 present the results obtained for the simulation of two well known benchmark cases, the 4:1 planar sudden contraction and the flow around a confined cylinder. For assessment purposes the results computed with the developed code will be compared with available data from the literature for the same case studies.

\subsection{Flow in a 4:1 planar sudden contraction}

A planar sudden contraction with contraction ratio $C R=H_{1} / H_{2}=4$ was chosen as the first test geometry (Fig. 3), because of the availability of numerical data in the literature [10]. The flow has a symmetry plane along the centerline $(y=0)$ for steady flow conditions, and to save computational resources and reduce the CPU times only half of the domain is considered. Fig. 3 shows the five structured blocks used to generate the five consecutively refined meshes (which are similar to those used in Alves et al. [10]), used to evaluate the order of convergence of the developed method and to obtain accurate results. The corner vortex size $x_{R}$, which will be used to estimate the accuracy of the developed code via the application of Richardson's extrapolation to the limit, was computed as the distance from the point where fluid velocity 
Table 1

Characteristics of the five meshes used for mesh convergence analysis in the 4:1 planar sudden contraction flow.

\begin{tabular}{|c|c|c|c|c|c|c|}
\hline \multirow[t]{2}{*}{ Block } & \multicolumn{3}{|l|}{ Mesh 1} & \multicolumn{3}{|l|}{ Mesh 2} \\
\hline & $\mathrm{NX} \times \mathrm{NY}$ & $f_{x}$ & $f_{y}$ & $\mathrm{NX} \times \mathrm{NY}$ & $f_{x}$ & $f_{y}$ \\
\hline Block I & $24 \times 10$ & 0.8210 & 0.8475 & $47 \times 20$ & 0.9061 & 0.9206 \\
\hline Block II & $24 \times 13$ & 0.8210 & 1.2091 & $47 \times 25$ & 0.9061 & 1.0996 \\
\hline Block III & $24 \times 5$ & 0.8210 & 0.7384 & $47 \times 9$ & 0.9061 & 0.8593 \\
\hline Block IV & $20 \times 10$ & 1.2179 & 0.8475 & $40 \times 20$ & 1.1036 & 0.9206 \\
\hline Block V & $7 \times 10$ & 1.3782 & 0.8475 & $13 \times 20$ & 1.1740 & 0.9206 \\
\hline NC & 942 & & & 3598 & & \\
\hline$\Delta x_{\min }=\Delta y_{\min }$ & $0.04 H_{2}$ & & & $0.02 \mathrm{H}_{2}$ & & \\
\hline \multirow[t]{2}{*}{ Block } & \multicolumn{3}{|l|}{ Mesh 3} & \multicolumn{3}{|l|}{ Mesh 4} \\
\hline & $\mathrm{NX} \times \mathrm{NY}$ & $f_{x}$ & $f_{y}$ & $\mathrm{NX} \times \mathrm{NY}$ & $f_{x}$ & $f_{y}$ \\
\hline Block I & $94 \times 40$ & 0.9519 & 0.9595 & $188 \times 80$ & 0.9756 & 0.9795 \\
\hline Block II & $94 \times 50$ & 0.9519 & 1.0486 & $188 \times 100$ & 0.9756 & 1.0240 \\
\hline Block III & $94 \times 17$ & 0.9519 & 0.9270 & $188 \times 34$ & 0.9756 & 0.9628 \\
\hline Block IV & $80 \times 40$ & 1.0505 & 0.9595 & $160 \times 80$ & 1.0249 & 0.9795 \\
\hline Block V & $25 \times 40$ & 1.0835 & 0.9595 & $50 \times 80$ & 1.0409 & 0.9795 \\
\hline $\mathrm{NC}$ & 14258 & & & 57032 & & \\
\hline$\Delta x_{\min }=\Delta y_{\min }$ & $0.01 H_{2}$ & & & $0.005 \mathrm{H}_{2}$ & & \\
\hline \multirow[t]{2}{*}{ Block } & \multicolumn{3}{|l|}{ Mesh 5} & & & \\
\hline & $\mathrm{NX} \times \mathrm{NY}$ & $f_{x}$ & $f_{y}$ & & & \\
\hline Block I & $376 \times 160$ & 0.9877 & 0.9897 & & & \\
\hline Block II & $376 \times 200$ & 0.9877 & 1.0119 & & & \\
\hline Block III & $376 \times 68$ & 0.9877 & 0.9812 & & & \\
\hline Block IV & $320 \times 160$ & 1.0124 & 0.9897 & & & \\
\hline Block V & $100 \times 160$ & 1.0202 & 0.9897 & & & \\
\hline NC & 228128 & & & & & \\
\hline$\Delta x_{\min }=\Delta y_{\min }$ & $0.0025 \mathrm{H}_{2}$ & & & & & \\
\hline
\end{tabular}

NX and NY are the number of cells along $x$ and $y$ directions, respectively, inside each block. $f_{x}$ and $f_{y}$ are expansion/contraction ratios inside each block.

$\mathrm{NC}$ is the number of cells for each mesh.

$\Delta x_{\min }$ and $\Delta y_{\min }$ are minimum cell size in each direction.

change direction to the corner point in Block III, measured on the last row of cells of this block (the cells near the north wall). Blocks I to III are in the upstream channel and Blocks IV and V in the downstream channel. The transition between Blocks IV and V occurs at $x=10 \mathrm{H}_{2}$ and the transition between Blocks II and III occurs at $y=3 \mathrm{H}_{2}$. The characteristics of the five hexahedral meshes used are presented in Table 1 and the region near the contraction of the most refined mesh (Mesh 5) is shown in Fig. 4. All meshes were designed with higher refinement near the walls and in the contraction region, as illustrated in Fig. 4 for Mesh 5 , because these two regions are known to present the highest gradients of the computed flow variables. The expansion or contraction geometrical factors are defined for each direction as the ratio of two consecutive cells lengths $\left(f_{x}=\Delta x_{i+1} / \Delta x_{i}\right.$ with $\Delta x_{i}$ being the length of cell $i$ in the $x$-direction). In this way, since $f_{x}>1$ in Block $\mathrm{V}$ (see Table 1), in the $x$ direction the cells expand from left to right. With this procedure, the minimum normalized cell size at the corner was $\Delta x_{\min } / H_{2}=\Delta y_{\min } / H_{2}=0.0025$ for the finest mesh, in Mesh 5. For each mesh refinement the number of cells along each direction (NX and NY) was doubled, and the corresponding expansion/contraction ratios $\left(f_{x}\right.$ and $f_{y}$ ) inside each sub-block were root-squared. We emphasize the large number of cells (NC) used in Mesh 5, a total of 228128 cells, which is one of the finest meshes used so far for this benchmark problem.

The computational domain spans from $x=-20 \mathrm{H}_{2}$ to $x=50 \mathrm{H}_{2}$ which is sufficiently large to avoid the effect of the inlet/outlet boundary conditions in the flow field in the contraction region, for the range of Deborah numbers simulated.

The Reynolds and Deborah numbers, used to characterize specific runs are defined on the basis of downstream channel variables (average velocity $U_{2}$ and channel height $H_{2}$ ):

$R e=\frac{\rho U_{2} H_{2}}{\eta_{P}}$

$D e=\frac{\lambda U_{2}}{H_{2}}$

The Reynolds number was fixed at 0.01 (representative of creeping flow) while $D e$ was varied from $D e=0$ (Newtonian fluid flow) up to $D e=5$. In all calculations, the constant $\eta^{\star}$ in Eq. (6) was set equal to the polymer viscosity coefficient, $\eta_{P}$. The incompressible steady-state solver simpleFoam, already present in the OpenFOAM computational library, was used for the Newtonian calculations. The Newtonian stress was discretized using the same scheme used for the implicit diffusive term in the iBSD technique developed for viscoelastic fluid flows. The Newtonian viscosity was assumed to be equal to the polymer viscosity $\left(\eta_{P}\right)$ of the UCM constitutive model.

The following boundary conditions were used for all the runs performed:

- for velocity, no-slip at the walls, symmetry at the centerline, fullydeveloped profile at the inlet [33] (with average velocity $U_{1}=U_{2} / C R$ ), and a zero gradient condition at the outlet, i.e., assuming fully-developed flow;

- for pressure, the inlet and wall boundary conditions were set as zero gradient, the centerline as symmetry boundary condition and at the outlet Dirichlet boundary condition was used, with a fixed value $p=0$. Notice that, although the zero pressure gradient specified at the inlet does not match with the fully developed Poiseuille flow with the average velocity $U_{1}$, this inconsistency does not affect the

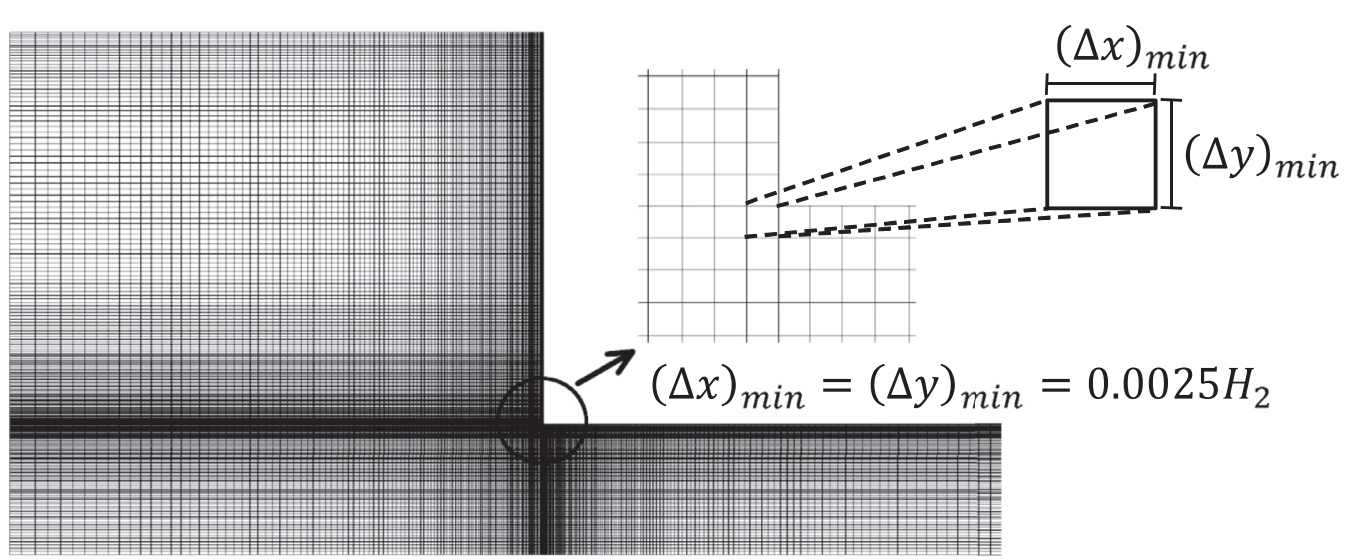

Fig. 4. Finest mesh used in the 4:1 planar contraction case study (Mesh 5). 
results, because the length of the upstream channel is sufficiently large to achieve fully developed flow conditions;

- finally, for the stress tensor, at the inlet a fully-developed profile was assumed [33], at the centerline a symmetry boundary condition was used, at the walls a linear extrapolation of the extra-stress components to the boundary was used and at the outlet a zero gradient condition was imposed.

All fields were set to zero at the initial time.

The use of a normalized time-step $\Delta t /\left(H_{2} / U_{2}\right)$ of $10^{-4}$ allowed to obtain converged solutions for all the runs performed. The maximum local Courant number corresponding to the normalized time-step $10^{-4}$, obtained for the 4:1 planar sudden contraction was 0.07. The MINMOD scheme was used in the discretization of the advective terms.

\subsubsection{Asymptotic behavior near the re-entrant corner}

First we investigated the asymptotic behavior near the re-entrant corner. For Newtonian fluids, it is known $[34,35]$ that the variation of velocity and stress components at that location follows the asymptotic expressions given by:

$\mathrm{u}_{i} \propto r^{0.545}$

$\tau_{i j} \propto r^{-0.455}$

for any given angle $\theta$ in the polar co-ordinates $(r, \theta)$ centered at the reentrant corner (see Fig. 3). For the assumed creeping flow conditions ( $R e=0.01$ ), the results obtained for $\theta=\pi / 2$ near the re-entrant corner are shown in Fig. 5. The velocity components are normalized with $U_{2}$ and the stress components with $\tau_{w}=3 \eta_{P} U_{2} / H_{2}$ (for Newtonian fluid flows $\eta_{P}$ is the Newtonian viscosity). In general it can be stated that the asymptotic behavior is well matched for all the five meshes except for the $\mathrm{u}_{\theta}$ and $\tau_{r \theta}$, which shows noticeable differences relatively to the slope in the coarser meshes. Similar differences were reported by Alves et al. [10] in their results.

In case of viscoelastic fluid flows described by the Oldroyd-B model, Hinch's analysis [36] derived the following asymptotic behavior near the re-entrant corner:

$\mathrm{u}_{i} \propto r^{5 / 9}$

$\tau_{i j} \propto r^{-2 / 3}$

for low Deborah number flows. As stated by Hinch [36], in the corner region the $r^{-2 / 3}$ elastic stress growth dominated the $r^{-4 / 9}$ solvent stress, so that in a sense the analysis is also adequate for a UCM fluid. Hence, the asymptotic expressions given by Eqs. (15) and (16) are also expected to hold for the UCM model. Thus, the developed numerical code was first tested for $D e=1$. The asymptotic behavior for velocity and stress components near the re-entrant corner, together with the theoretical asymptotes of Hinch [36], are shown in Fig. 6, for different values of the angle $(\theta)$. The predicted asymptotic stress behavior agrees with Hinch's analysis. The larger differences found next to the wall are expected since, as Hinch predicted, the stress growths with a power law trend $r^{-2 / 3}$ is only valid in the corner region but far from the walls. The similarity solution of Hinch is of the form $Q f(\theta) r^{-\alpha}(Q$ is an amplitude determined by conditions away from the corner and $f$ has a sinusoidal structure [36]) both for $\mathbf{u}$ and $\tau$ and, therefore, in a log-log plot the effect of varying $\theta$ is to shift the straight lines but keeping the same slope $(-\alpha)$ [37].

The effect of De number on the asymptotic predictions is shown in Fig. 7, by comparing the results obtained for $D e=0.03,1$ and 5. The velocity components and the normal stress $\tau_{r r}$ maintain the slope for all De, $5 / 9$ and $-2 / 3$, respectively. However, the other normal $\left(\tau_{\theta \theta}\right)$ and shear $\left(\tau_{r \theta}\right)$ stresses present a different trend at lower $D e$, which follows very well the, referred above, slope $-4 / 9$ corresponding to the solvent stress in the core region. Similar results were obtained by Alves et al. [37].

\subsubsection{Streamlines and corner vortex size}

In this section we analyze the flow patterns (streamline plots) predicted in the different meshes as a function of $D e$. The code developed was able to produce converged solutions up to $D e=5$ in Mesh 5 (the maximum value tested in this work) extending the results of Alves et al. [10] using the MINMOD scheme.

Fig. 8 presents a comparison between the streamlines predicted, with our implementation in OpenFOAM ${ }^{\oplus}$ and the results of Alves et al. [10], in Mesh 3, with the MINMOD scheme and for De numbers between 0 to 5 . The results obtained with the developed code follow the same trend as in Alves et al. [10], showing a decrease of the corner vortex as $D e$ is increased, up to $D e=3$. In addition, a small lip vortex can be seen at $D e=2$, which increases in size with the increase of $D e$ and subsequently merges with the salient corner vortex to become dominant. The onset of the vortex merging and the increase of the lip vortex with $D e$ is highly dependent on the mesh resolution, as also found recently by Pimenta and Alves [16] for an Oldroyd-B model.

The quantitative comparison of the corner vortex is made measuring its dimensionless length $X_{R}=x_{R} / H_{2}$ (see Fig. 3). The mesh refinement used allowed to apply Richardson's extrapolation for the $X_{R}$ variable using the three finest meshes. Table 2 compares the results obtained with the developed code and the data in Alves et al. [10]. The $X_{R}$ results in the most refined mesh (Mesh 5), obtained with the developed code, are within $0.6 \%$ from the extrapolated values.

The predicted flow pattern at moderate and high $D e$, respectively $D e=3$ and $D e=5$, was found to be more sensitive to mesh refinement, as shown in Fig. 9 for Meshes 2-5. As De is increased, the mesh refinement level required to capture accurately the size and shape of the corner vortex also needs to be higher. For instance, Mesh 3 has a refinement that seems to be enough to estimate, accurately, the corner vortex size up to $D e=3$; however, for $D e=5$ a more refined mesh is needed (Mesh 4), as shown in Table 2 and the streamline plots shown in Fig. 9.

As discussed in Roache [38], Richardson extrapolation can be applied both to point-wise values or to solution functionals, when consistent or high-order methods are used in their calculation. In the contraction flow simulation, the corner vortex size $X_{R}$ is an adequate and often used solution functional, which is highly sensitive to mesh refinement. The corner vortex size, $X_{R}$, was used to quantitatively estimate the uncertainty of the numerical results using Richardson extrapolation. Fig. 10 shows the dependence of the estimated error for $X_{R}$ (assuming the extrapolated values are the correct ones) on the mesh size, for all $D e$ tested. Although second order schemes were used to discretize all the governing equation terms, as can be seen on the figure, the method used has an order of convergence between the first-order and the second-order for all De tested. A similar problem, concerning the degradation of the convergence order, was identified in Comminal et al. [39], when the linear interpolation was used for the stress terms, as happens in this work for the $\nabla \cdot \tau_{P}$ in the momentum conservation equation (Eq. (6)).

\subsubsection{Distribution of velocity and first-normal stress difference near the downstream wall and along the centerline}

The distribution of the streamwise velocity component and the first normal stress difference along the line $y / H_{2}=0.98$ is illustrated in Fig. 11 for three cases, $D e=\{0,3,5\}$. The figure shows that the first normal stress difference, $N_{1}=\tau_{x x}-\tau_{y y}$, is more sensitive to mesh refinement than the streamwise velocity along the line $y / H_{2}=0.98$, due to the singular behavior of $N_{1}$ at the re-entrant corner. In addition, as $D e$ is increased the results become more sensitive to mesh refinement, which induces a non-monotonic variation of $N_{1}$.

The predicted centerline profiles for the longitudinal velocity and $N_{1}$ at the same Deborah numbers are shown in Fig. 12. As expected, the results obtained are now less sensitive to mesh refinement. 


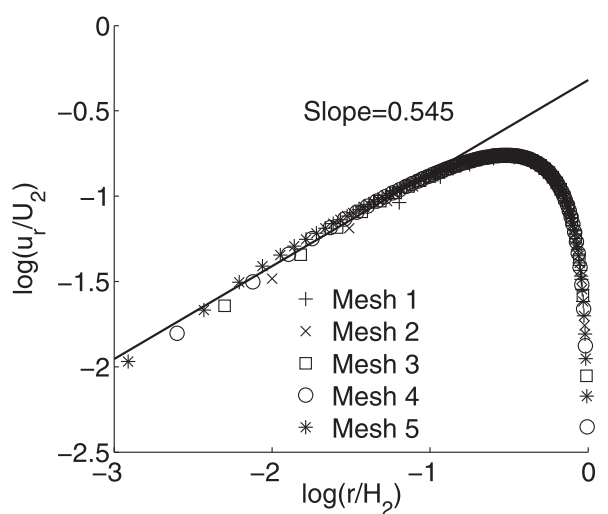

(a)

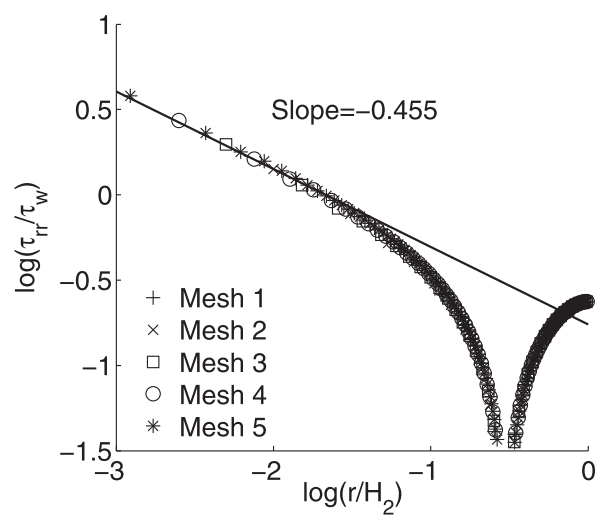

(c)

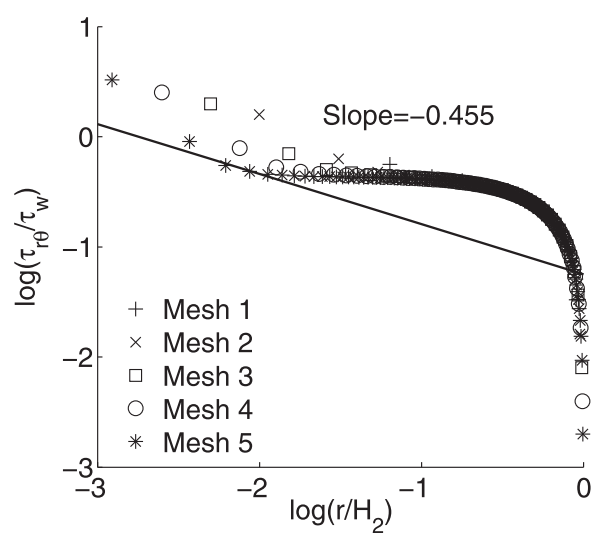

(e)

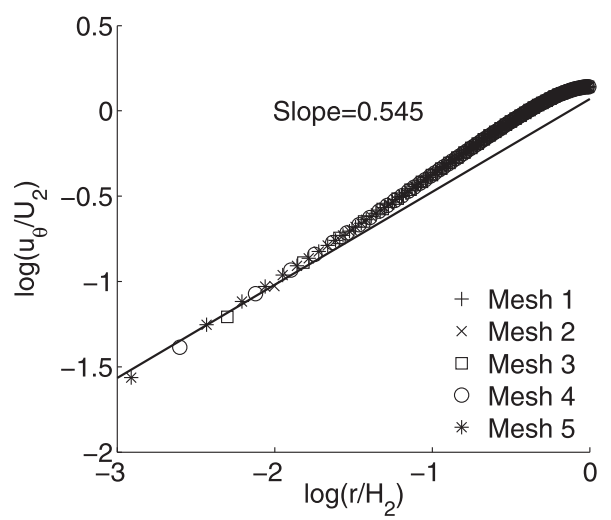

(b)

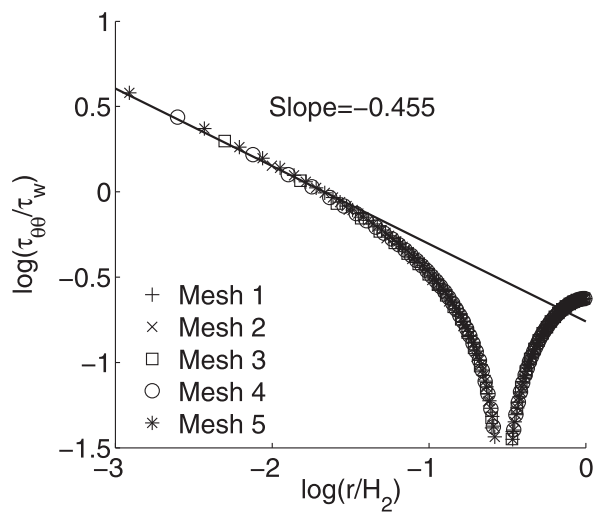

(d)
Fig. 5. Asymptotic behavior of the predicted velocity and stress components near the re-entrant corner, for a Newtonian fluid along direction $\theta=\pi / 2$ (see Fig. 3).

\subsection{Flow around a confined cylinder}

The second case study refers to the planar flow past a confined circular cylinder placed at the centerline of a channel (see Fig. 13). The blockage ratio in this study, defined as the ratio of cylinder radius $R$ to channel half-height $h$, is $\beta=0.5$. The purpose of choosing this problem is to test the capability of the code to achieve accurate results both in Newtonian and viscoelastic fluid flows, when using non-orthogonal meshes.

The computational domain extends from $x=-20 R$, where a fully developed profile is imposed with average velocity $U$, up to $x=60 R$, which is sufficiently long to achieve fully-developed flow conditions at the exit. The flow has a symmetry plane along the centerline $(y=0)$ and to save computational resources and reduce the CPU times, only half of the domain is considered. The boundary conditions used for this case study are:
- at the inlet $(x / R=-20)$, Dirichlet conditions based on fully developed profiles for velocity and stress components, and zero-gradient for pressure. Notice again that the pressure-gradient of the fully developed Poiseuille flow should have been used to avoid inconsistencies, but that does not affect the results as the length of the channel entrance is long enough to achieve fully developed conditions;

- at the outlet $(x / R=60)$, zero Neumann boundary conditions for the velocities and the stress components are applied, and the pressure value is fixed, $p=0$;

- at the channel wall $(y=h)$ and at the cylinder surface $(r=R)$, we applied a no-slip boundary condition to the velocity components and a zero gradient for pressure, while the stress tensor components were linearly extrapolated;

- at the symmetry plane $(y=0)$, symmetry conditions are imposed. 


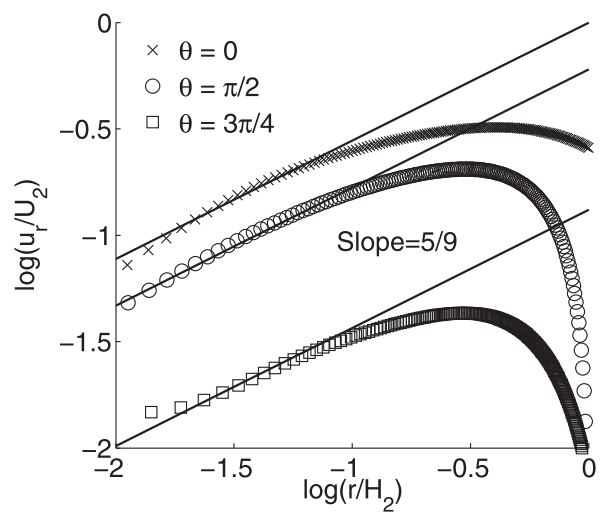

(a)

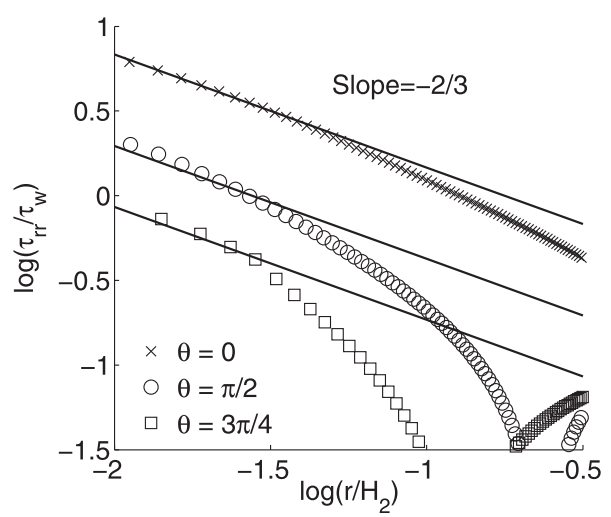

(c)

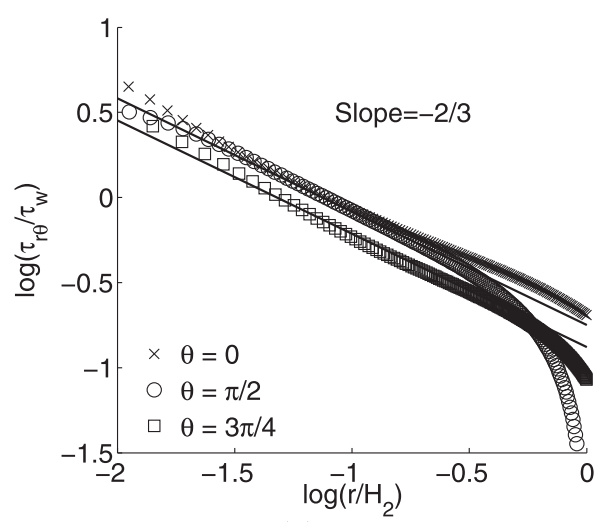

(e)

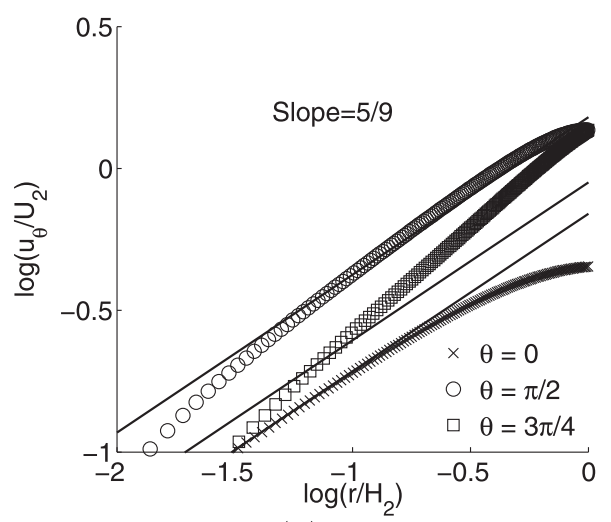

(b)

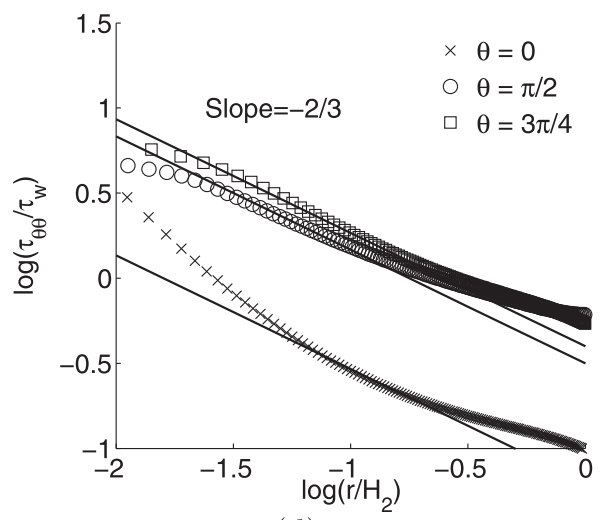

(d)
Fig. 6. Asymptotic behavior of the predicted velocity and stress components near the re-entrant corner, for a viscoelastic UCM fluid at $D e=1$, along directions $\theta=\{0, \pi / 2,3 \pi / 4\}$ (see Fig. 3 ).
For the generation of the block-structured meshes eight blocks were used, as represented in Fig. 13. The meshes were generated with a higher density of cells near the cylinder surface in order to resolve accurately the boundary-layer, where higher stress gradients are expected. Four meshes with different degrees of refinement were used for the mesh convergence analysis, and are presented in Table 3. The numbers in the mesh names indicate the number of radial cells between the cylinder surface and the channel wall. Meshes M30, M60 and M120 were also used in Alves et al. [11]. The refined mesh M240 is shown in Fig. 14. We emphasize the large number of cells used in this mesh, a total of 278 400, which is one of the finest meshes used so far in this benchmark problem.

The Reynolds and Deborah numbers are defined for this problem based on the inlet average velocity, $U$, and the cylinder radius, $R$ :

$R e=\frac{\rho U R}{\eta_{P}}$
$D e=\frac{\lambda U}{R}$

and $R e$ was fixed at 0.01 (representative of creeping flow) while $D e$ was varied in the range 0 to 0.8 . Similarly to what was done in the $4: 1$ planar sudden contraction flow case study, the parameter $\eta^{\star}$ in Eq. (6) was considered equal to the polymer viscosity, $\eta^{\text {殅 }}=\eta_{P}$.

The use of a normalized time-step $\Delta t /(R / U)$ of $10^{-4}$ allowed to obtain converged solutions for all the runs performed. The maximum local Courant number corresponding to the normalized time-step $10^{-4}$, obtained for the flow around a confined cylinder was 0.3. The SMART scheme was used in the discretization of the advective terms.

\subsubsection{Drag coefficient}

The dimensionless drag coefficient $C_{D}$, resulting from the surface integration of the stress and pressure fields around the cylinder, was computed as: 


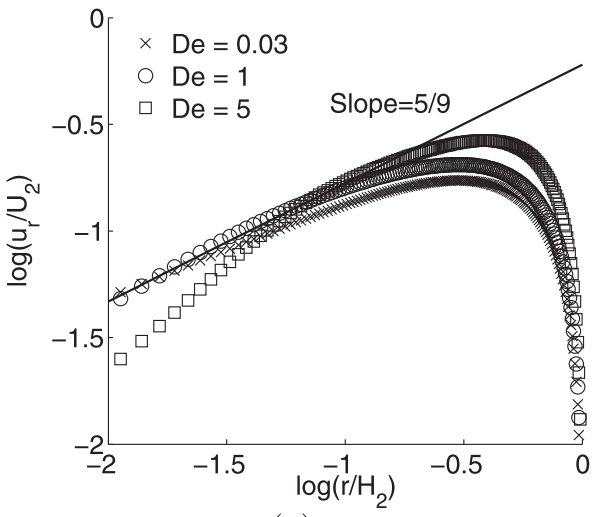

(a)

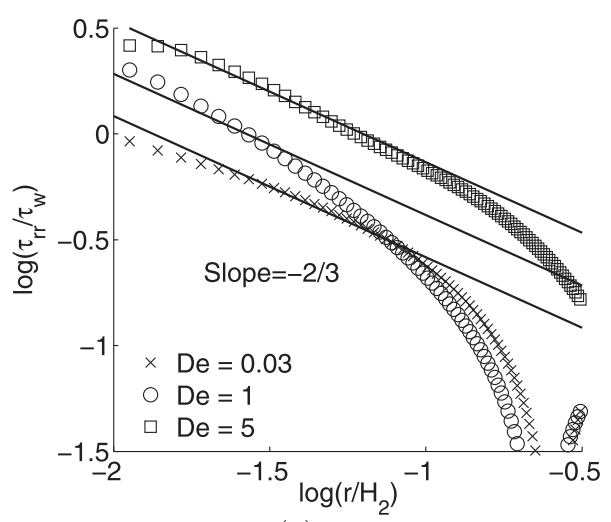

(c)

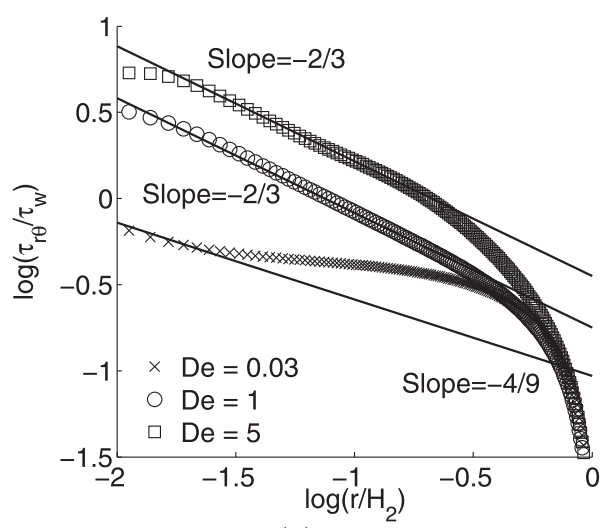

(e)

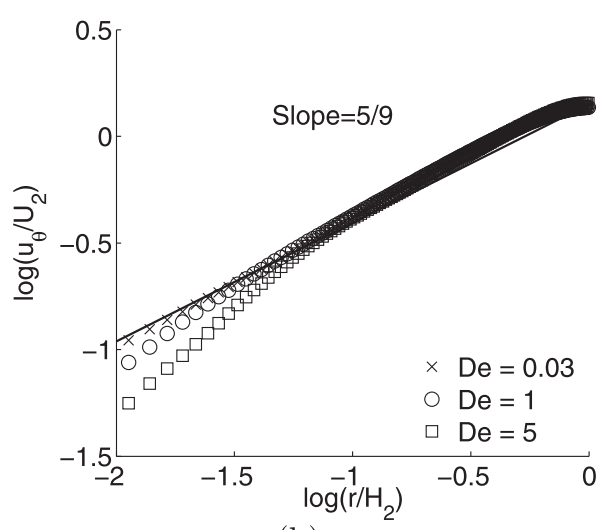

(b)

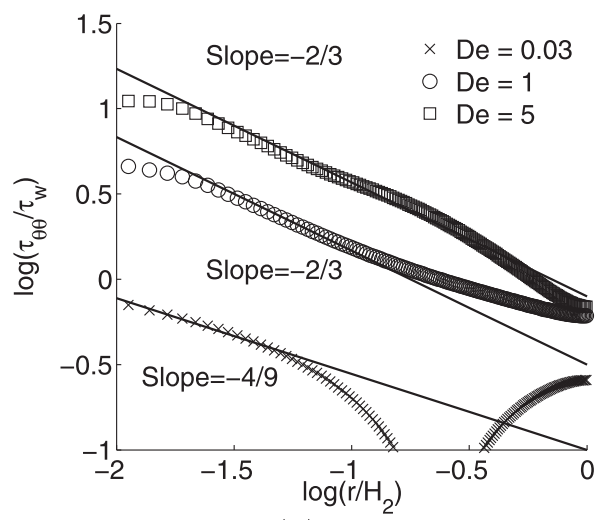

(d)
Fig. 7. Effect of the De number on the asymptotic behavior $(\theta=\pi / 2)$. 


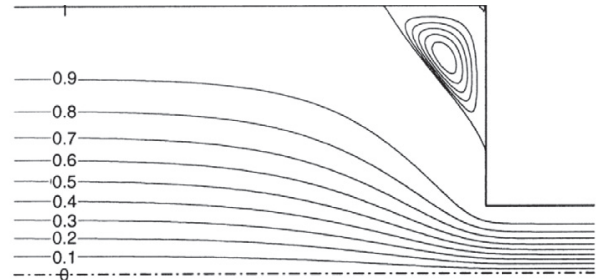

(a) $D e=0$

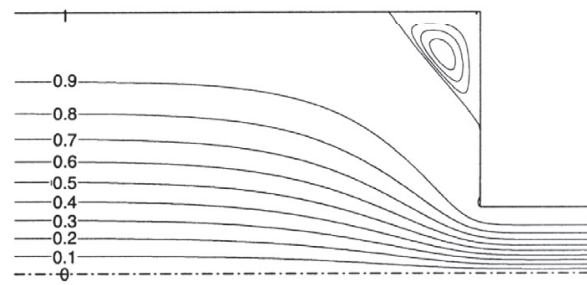

(c) $D e=1$

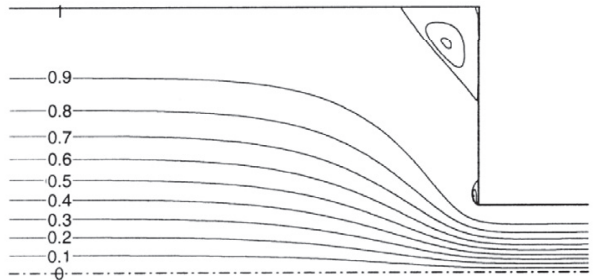

(e) $D e=2$

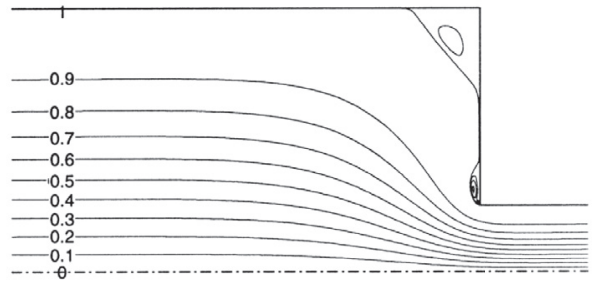

(g) $D e=3$

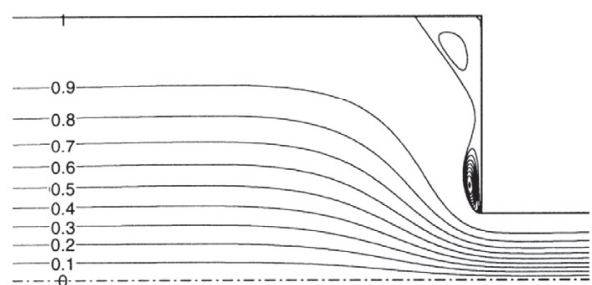

(i) $D e=4$

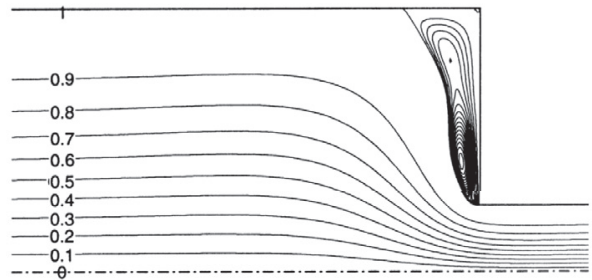

(k) $D e=5$

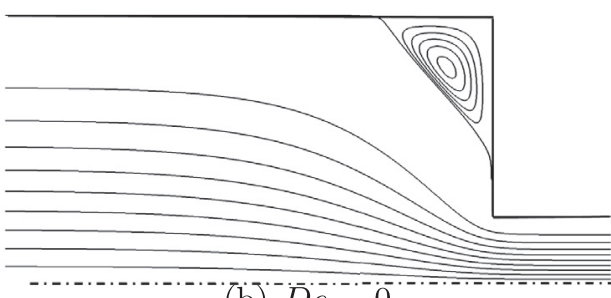

(b) $D e=0$

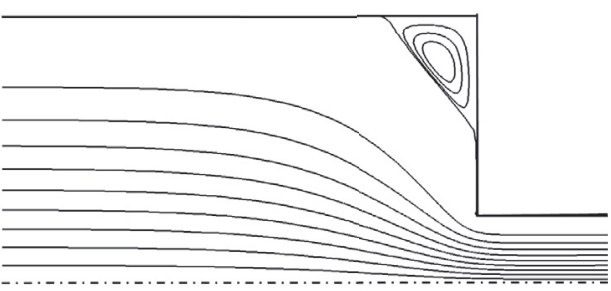

(d) $D e=1$

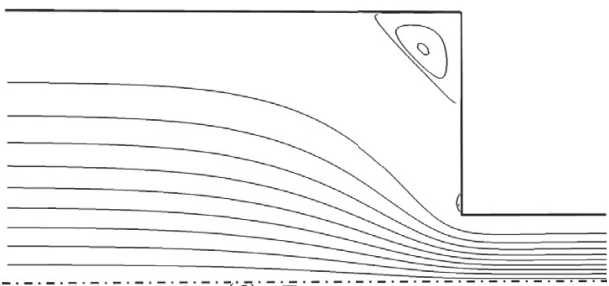

(f) $D e=2$

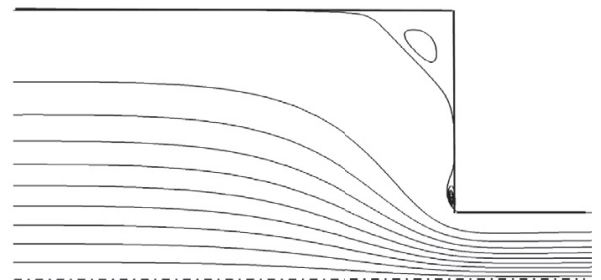

(h) $D e=3$

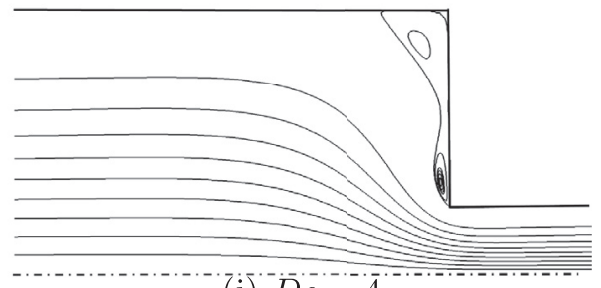

(j) $\bar{D} e=4$

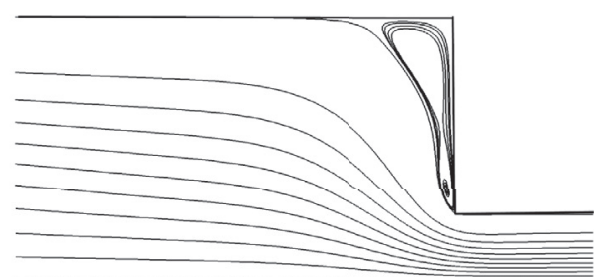

(1) $D e=5$
Fig. 8. Streamlines obtained with Mesh 3, for $D e=\{0,1,2,3,4,5\}$ at $R e=0.01$. Left: results obtained by Alves et al. [10]; right: results obtained with developed code in OpenFOAM ${ }^{\circ}$. 
Table 2

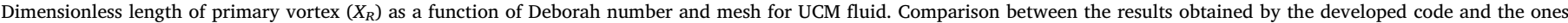
obtained by Alves et al. [10].

\section{Developed code in OpenFOAM}

\begin{tabular}{|c|c|c|c|c|c|c|c|}
\hline$D e$ & Mesh 1 & Mesh 2 & Mesh 3 & Mesh 4 & Mesh 5 & Extrapolated & Difference $(\%)^{\mathrm{a}}$ \\
\hline 0 & 1.438 & 1.479 & 1.492 & 1.495 & 1.496 & 1.4965 & 0.03 \\
\hline 1 & 1.293 & 1.336 & 1.326 & 1.32711 & 1.32696 & 1.32694 & 0.002 \\
\hline 2 & 1.207 & 1.200 & 1.130 & 1.101 & 1.091 & 1.0857 & 0.5 \\
\hline 3 & 1.333 & 1.118 & 0.958 & 0.900 & 0.885 & 0.880 & 0.6 \\
\hline 4 & 1.391 & 1.088 & 0.845 & 0.75 & 0.732 & 0.728 & 0.6 \\
\hline 5 & 1.469 & 1.101 & 0.758 & 0.636 & 0.617 & 0.613 & 0.6 \\
\hline \multicolumn{8}{|c|}{ Alves et al. [10] } \\
\hline$D e$ & Mesh 1 & Mesh 2 & Mesh 3 & Mesh 4 & Extrapolated & Difference $(\%)^{\mathrm{b}}$ & \\
\hline 0 & 1.472 & 1.488 & 1.494 & 1.495 & 1.496 & 0.1 & \\
\hline 1 & 1.349 & 1.371 & 1.349 & 1.339 & 1.335 & 0.3 & \\
\hline 2 & 1.631 & 1.259 & 1.154 & 1.118 & 1.105 & 1.2 & \\
\hline 3 & 1.517 & 1.266 & 1.014 & 0.946 & 0.923 & 2.5 & \\
\hline 4 & 1.644 & 1.337 & 0.987 & c & 0.87 & 13 & \\
\hline 5 & 1.687 & 1.517 & 1.127 & c & 0.997 & 13 & \\
\hline
\end{tabular}

${ }^{\text {a }}$ Calculated between Mesh 5 and extrapolated values.

b Calculated between Mesh 4 (or 3) and extrapolated values.

c Convergence criterion not attained (solution oscillates).

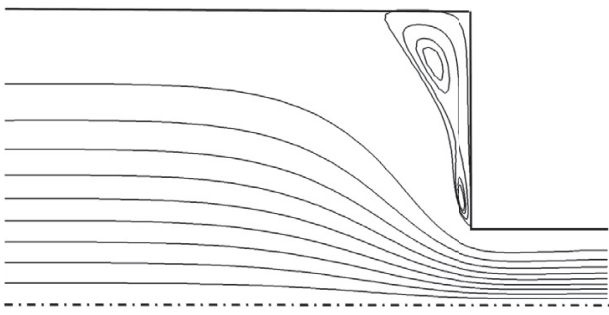

(a) Mesh 2

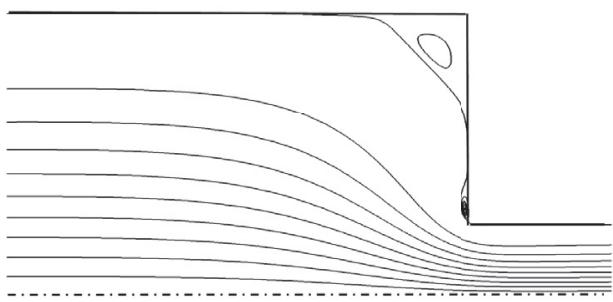

(c) Mesh 3

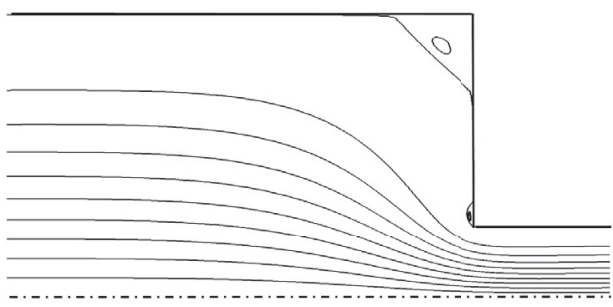

(e) Mesh 4

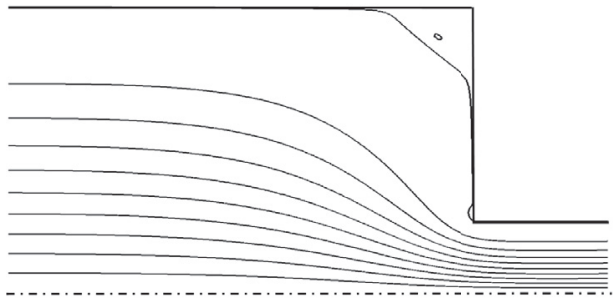

(g) Mesh 5

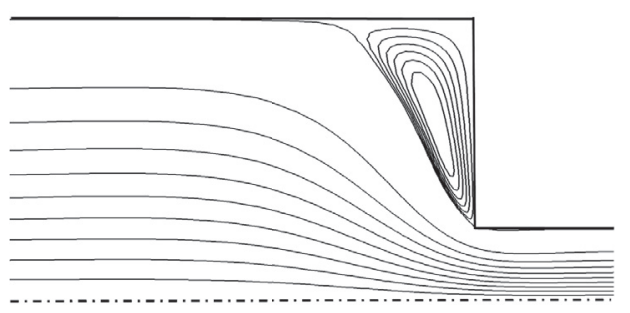

(b) Mesh 2

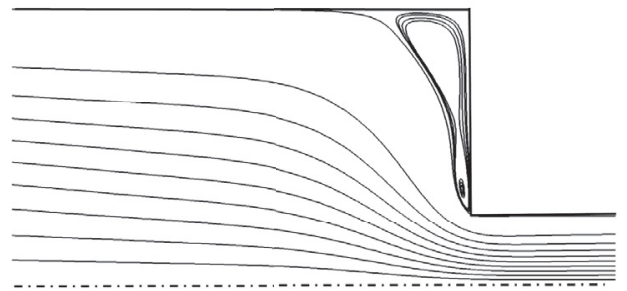

(d) Mesh 3

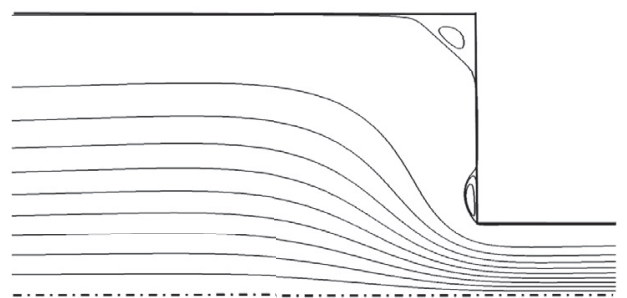

(f) Mesh 4

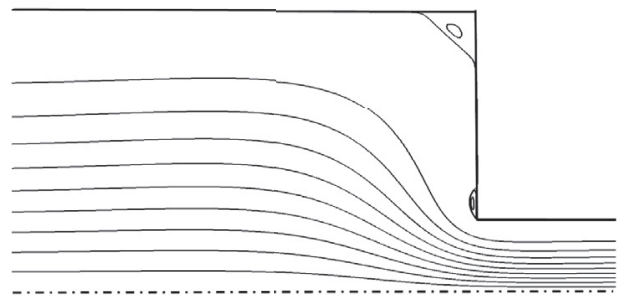

(h) Mesh 5
Fig. 9. Effect of mesh refinement in the streamline patterns for $R e=0.01$ at moderate and high Deborah numbers, $D e=3$ (left) and $D e=5$ (right), respectively. 


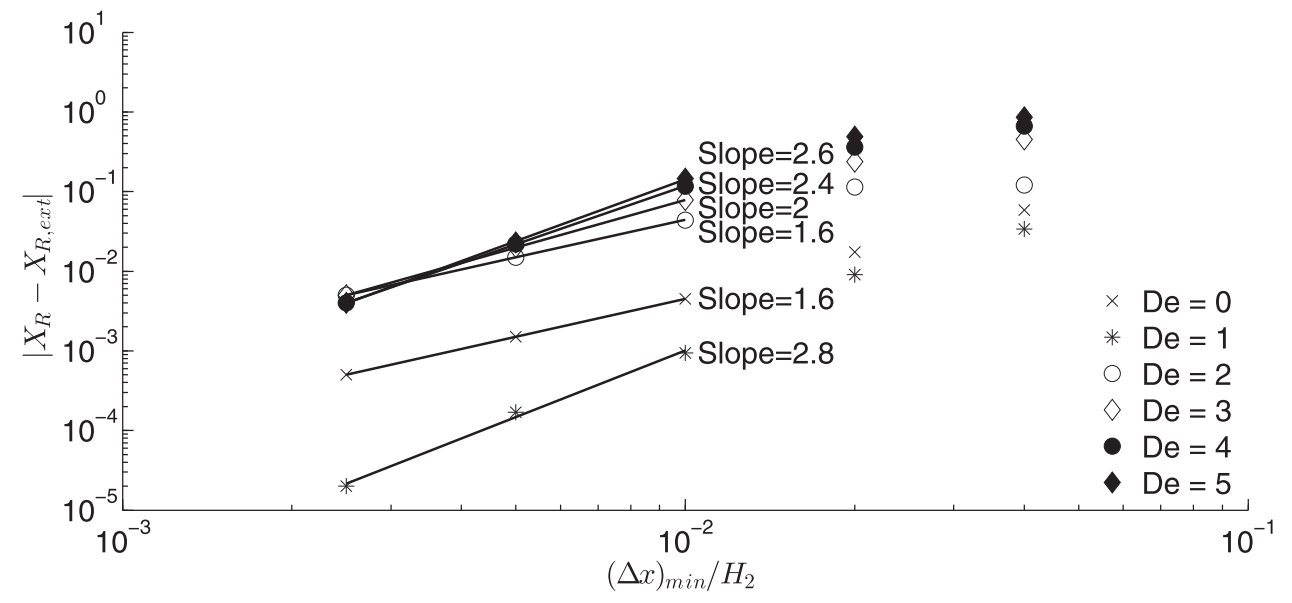

Fig. 10. Estimated error for $X_{R}$ as a function of mesh refinement and De for the UCM fluid in the $4: 1$ planar sudden contraction flow.
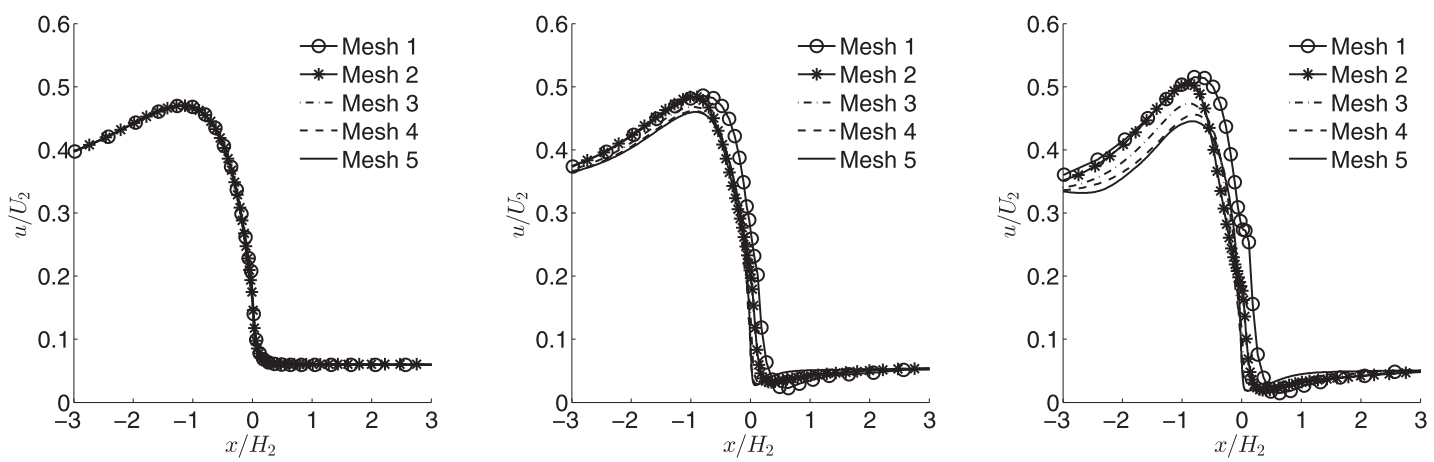

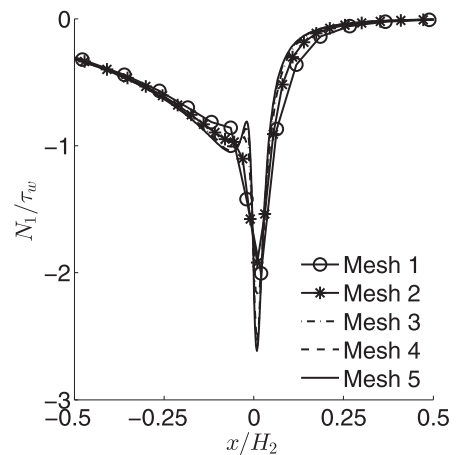

(a)

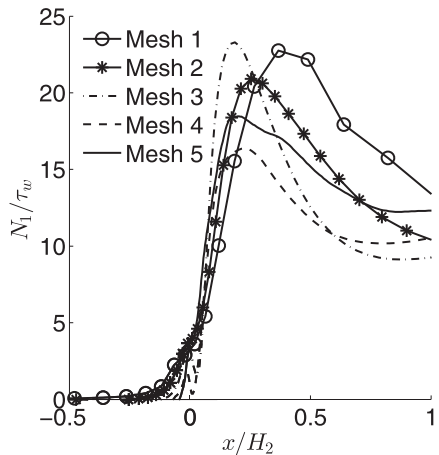

(b)

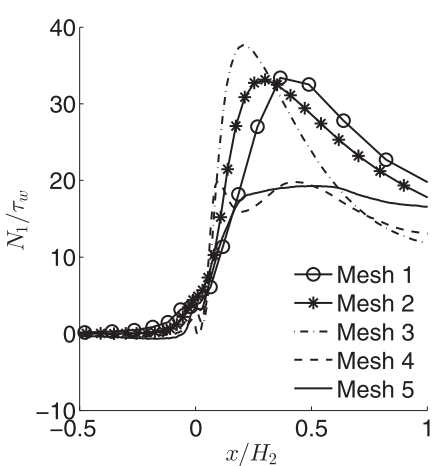

(c)

Fig. 11. Predicted streamwise velocity and first normal stress difference profiles at $y / H_{2}=0.98$ for (a) $D e=0$, (b) $D e=3$ and (c) $D e=5$.

are shown in Fig. 17. This evolution presents two maxima, the first one is within the thin boundary layer over the cylinder surface and the second maximum occurs in the wake centerline, downstream of the rear stagnation point. The results obtained with the developed code show a similar tendency of the ones obtained by Alves et al. [11]. Namely, as the $D e$ increases the slope of the maximum stress decreases at the cylinder surface and increases at the wake.

\section{Conclusions}

A general finite-volume methodology for the computation of the flow of viscoelastic fluids described by differential-type constitutive equations was developed using the open-source OpenFOAM ${ }^{\circ}$ framework. The developed code can be applied to orthogonal or non-orthogonal meshes and was found to predict accurately steady-state solutions of the UCM fluid, which is known to be challenging from a numerical point of view.

An improved version of the both-sides diffusion technique (iBSD) was proposed and implemented numerically. The modification introduced was mainly related with the treatment of the additional diffusion term on the right-hand-side of the momentum equation.

The improved numerical solver was shown to assure the coupling between the velocity and stress fields, and thus provide physically sound predictions, which are difficult when dealing with constitutive models that do not consider the contribution of a solvent viscosity, as happens for the UCM. High resolution interpolation schemes were used for the discretization of the advective terms to enhance the results precision. The code accuracy was assessed with two widely used benchmark case studies: the 4:1 planar sudden contraction flow and the flow around a confined cylinder. In both cases, the simulations were performed at $R e=0.01$, representing creeping flow conditions. For the former case, the Deborah number varied from 0 to 5 and for the flow past a confined cylinder, $D e$ was varied from 0 to 0.8 .

Additionally, for the contraction flow case study, finer meshes were employed, when compared with the ones of Alves et al. [10], and it was possible to obtain converged solutions up to $D e=5$. Similarly, for the 

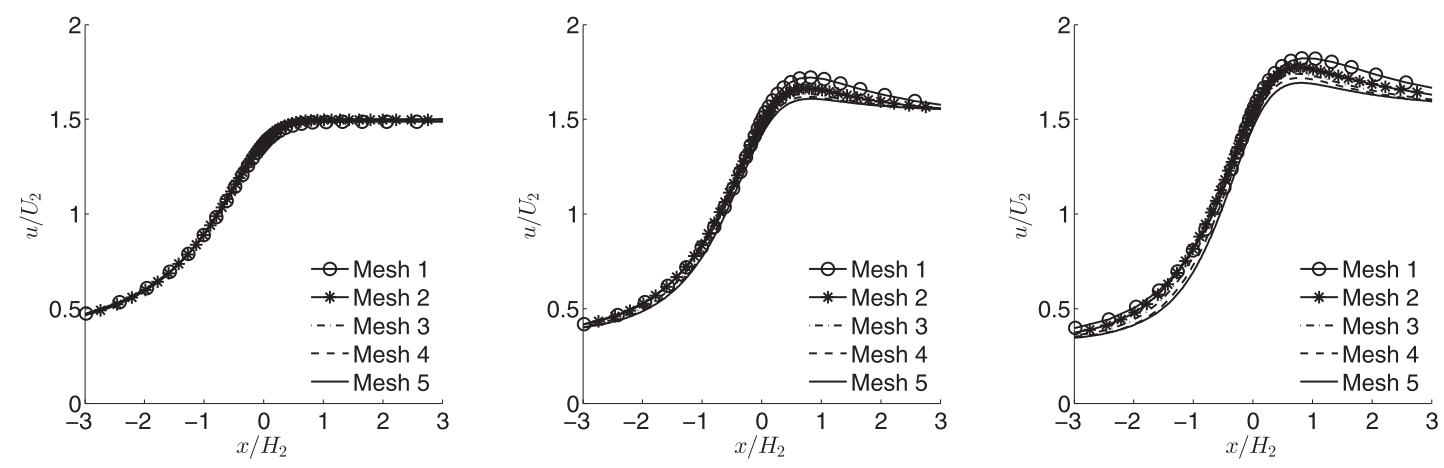

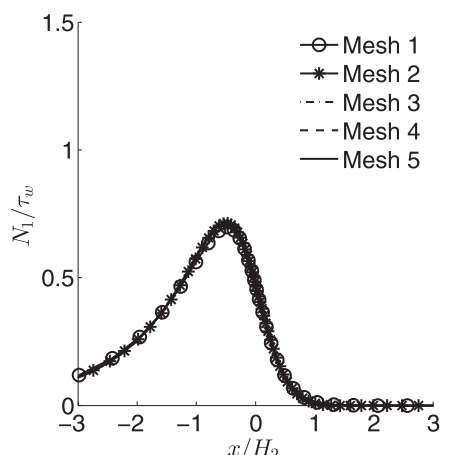

(a)

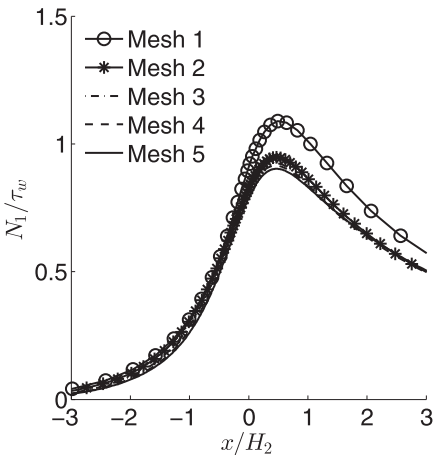

(b)

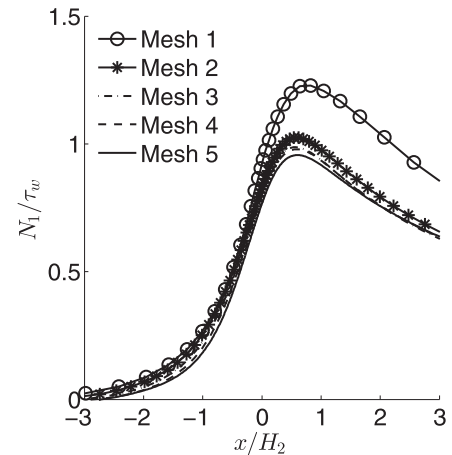

(c)

Fig. 12. Predicted streamwise velocity and first normal stress difference profiles at centerline $y / H_{2}=0$ for (a) $D e=0$, (b) $D e=3$ and (c) $D e=5$.

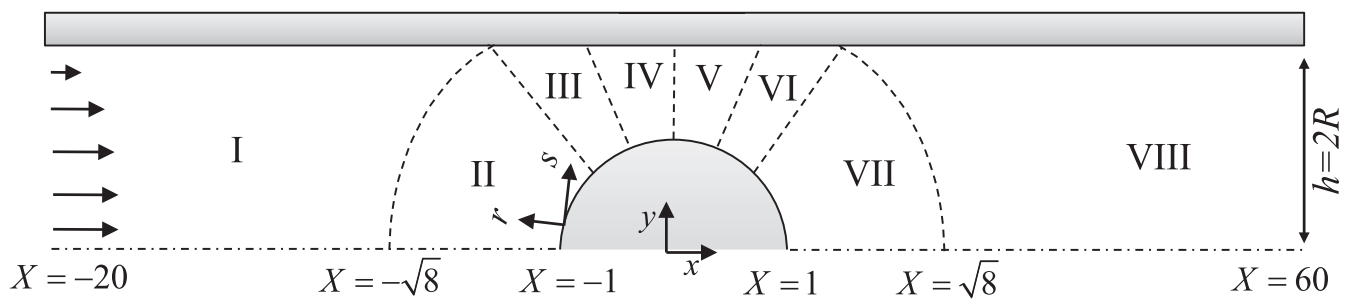

Fig. 13. Schematic representation of the flow past a circular cylinder. The roman numbering refers to the identification of the blocks used to generate the meshes $(X=x / R)$. The origin of the radial and tangential coordinate system $(r-s)$ is considered in $X=-1$ and the tangential coordinate, $s$, is computed as the length of a circular arc.

Table 3

Characteristics of the four meshes used for mesh convergence analysis in the flow around a confined cylinder.

\begin{tabular}{|c|c|c|c|c|c|c|c|c|}
\hline \multirow[t]{2}{*}{ Block } & \multicolumn{2}{|l|}{ M30 } & \multicolumn{2}{|l|}{ M60 } & \multicolumn{2}{|l|}{ M120 } & \multicolumn{2}{|l|}{ M240 } \\
\hline & $\mathrm{NS} \times \mathrm{NR}$ & $f_{r}$ & $\mathrm{NS} \times \mathrm{NR}$ & $f_{r}$ & $\mathrm{NS} \times \mathrm{NR}$ & $f_{r}$ & $\mathrm{NS} \times \mathrm{NR}$ & $f_{r}$ \\
\hline Block I & $25 \times 24$ & 1.1029 & $50 \times 48$ & 1.0502 & $100 \times 96$ & 1.0248 & $200 \times 192$ & 1.0124 \\
\hline Block II & $25 \times 30$ & 1.1075 & $50 \times 60$ & 1.0524 & $100 \times 120$ & 1.0259 & $200 \times 240$ & 1.0129 \\
\hline Block III & $13 \times 30$ & 1.1075 & $25 \times 60$ & 1.0524 & $50 \times 120$ & 1.0259 & $100 \times 240$ & 1.0129 \\
\hline Block IV & $13 \times 30$ & 1.1075 & $25 \times 60$ & 1.0524 & $50 \times 120$ & 1.0259 & $100 \times 240$ & 1.0129 \\
\hline Block V & $13 \times 30$ & 1.1075 & $25 \times 60$ & 1.0524 & $50 \times 120$ & 1.0259 & $100 \times 240$ & 1.0129 \\
\hline Block VI & $13 \times 30$ & 1.1075 & $25 \times 60$ & 1.0524 & $50 \times 120$ & 1.0259 & $100 \times 240$ & 1.0129 \\
\hline Block VII & $25 \times 30$ & 1.1075 & $50 \times 60$ & 1.0524 & $100 \times 120$ & 1.0259 & $200 \times 240$ & 1.0129 \\
\hline Block VIII & $25 \times 30$ & 1.1323 & $50 \times 60$ & 1.0641 & $100 \times 120$ & 1.0316 & $200 \times 240$ & 1.0157 \\
\hline NCV & 4410 & & 17400 & & 69600 & & 278400 & \\
\hline$(\mathrm{NS})_{\mathrm{tot}}$ & 102 & & 200 & & 400 & & 800 & \\
\hline$(\Delta r / R)_{\min }$ & 0.00963 & & 0.00481 & & 0.00238 & & 0.00119 & \\
\hline$(\Delta s / R)_{\min }$ & 0.0302 & & 0.0157 & & 0.00785 & & 0.003925 & \\
\hline
\end{tabular}

NS, NR: number of cells in tangential and radial directions.

NCV: total number of control volumes.

(NS) $)_{\text {tot }}$ : number of tangential cells around half-cylinder.

$f_{r}$ is expansion/contraction ratio inside each block in the radial direction. For the tangential direction the mesh is uniform.

$\Delta r$ and $\Delta s$ are the minimum cell size around the cylinder surface in the radial and tangential directions, respectively.

flow around a confined cylinder, meshes more refined than the ones of Alves et al. [11] were employed, and converged solutions were achieved up to $D e=0.8$. For both cases the method used has an order of accuracy between the first-order and the second-order for all $D e$ tested.
The results obtained in both test cases were accurately predicted in the sense that the vortex length size and drag coefficient show less than $0.6 \%$ and $0.08 \%$ differences, respectively, when comparing the finest mesh result and the extrapolated value. These results improve the 


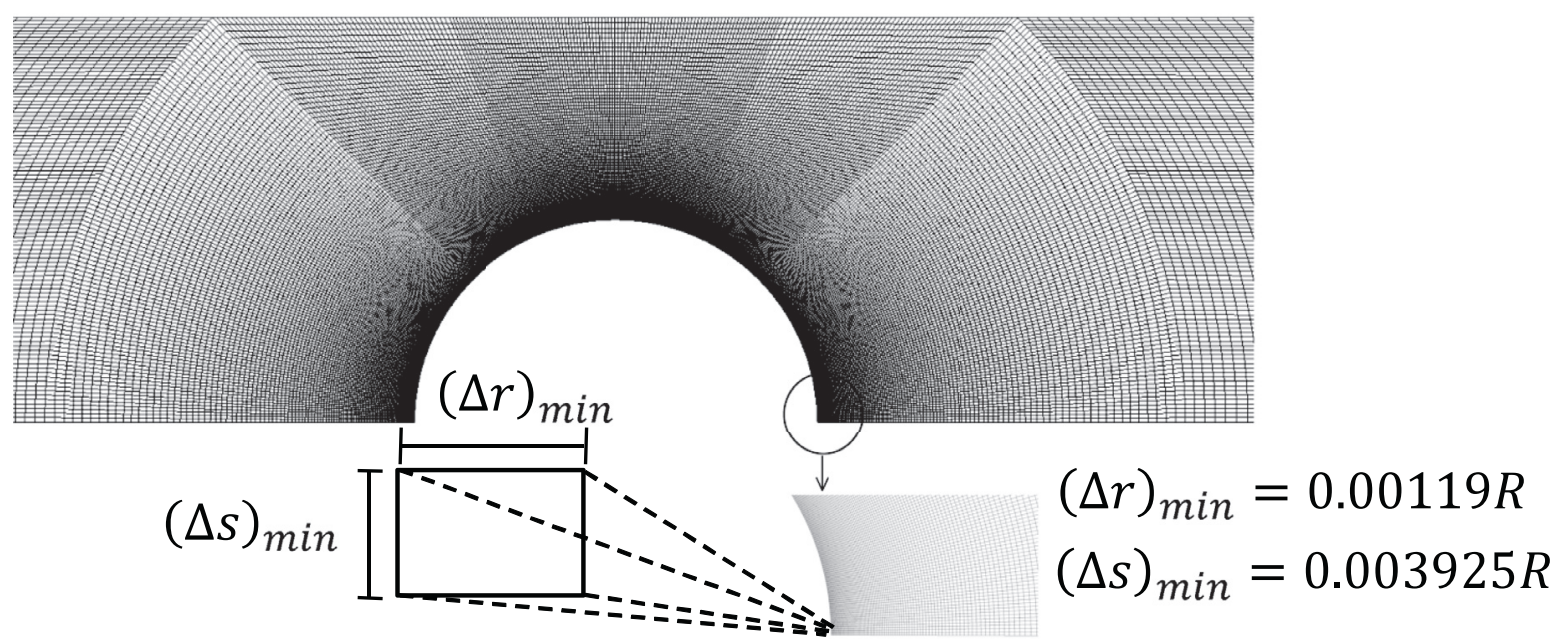

Fig. 14. Finest mesh used in the flow around a confined cylinder case study (M240).

Table 4

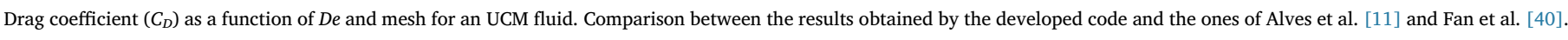

\begin{tabular}{|c|c|c|c|c|c|c|}
\hline \multicolumn{7}{|c|}{ Developed code } \\
\hline$D e$ & M30 & M60 & M120 & M240 & Extrapolated & Difference $(\%)^{\mathrm{a}}$ \\
\hline 0 & 131.998 & 132.397 & 132.484 & 132.500 & 132.504 & 0.003 \\
\hline 0.3 & 109.519 & 109.005 & 108.900 & 108.872 & 108.862 & 0.009 \\
\hline 0.6 & 94.027 & 92.962 & 92.703 & 92.625 & 92.591 & 0.04 \\
\hline 0.8 & 90.597 & 89.090 & 88.693 & 88.555 & 88.481 & 0.08 \\
\hline \multicolumn{5}{|c|}{ Alves et al. [11] } & \multicolumn{2}{|l|}{ Fan et al. [40] } \\
\hline$D e$ & M30 & M60 & M120 & Extrapolated & DEVSS & \\
\hline 0 & 132.23 & 132.342 & 132.369 & 132.378 & 132.36 & \\
\hline 0.3 & - & 108.515 & 108.614 & 108.647 & 108.68 & \\
\hline 0.6 & - & 92.277 & 92.298 & 92.305 & 92.37 & \\
\hline 0.8 & - & 88.253 & 88.178 & 88.153 & 88.18 & \\
\hline
\end{tabular}

${ }^{\text {a }}$ Calculated between M240 and extrapolated values.

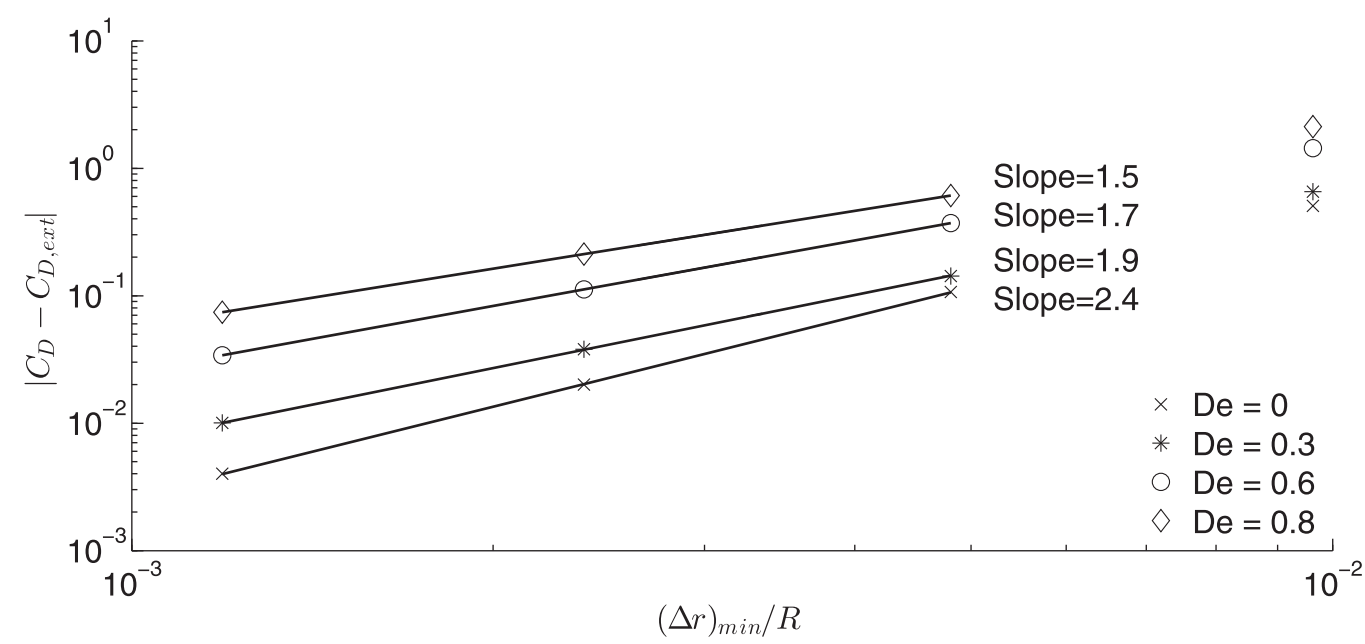

Fig. 15. Estimated error for $C_{D}$ as a function of mesh refinement and $D e$ number for the UCM flow around a confined cylinder case study.

accuracy of the data available in the literature for both benchmark case studies.

In summary, the results obtained show that the newly improved viscoelastic solver using an open-source code can predict accurately the flow patterns of the upper-convected Maxwell fluid in the two benchmark problems tested. Based on these achievements, the authors consider that it would be worth to test the iBSD approach with other viscoelastic models, differential or integral, which show similar numerical issues, but are in general easier to handle when compared with the UCM model. Moreover, the extension to transient cases is straightforward and is also worth to assess, just requiring additional iterations at each time step, to assure that the contributions of the additional diffusion terms, on the left and right side of the momentum conservation equations, are equal. 

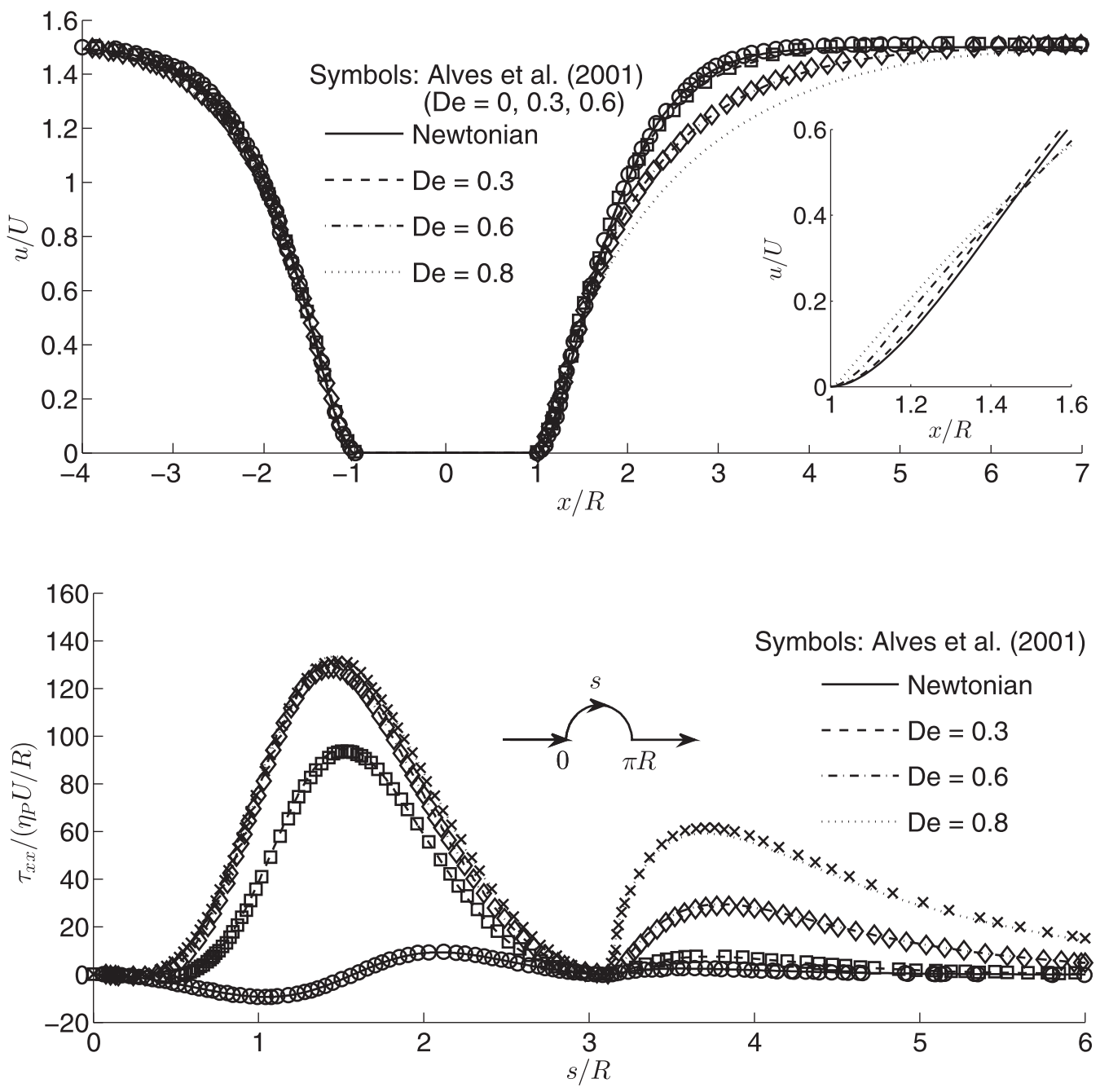

Fig. 16. Effect of $D e$ on the profiles of the longitudinal velocity along the centerline for the UCM fluid flow around a confined cylinder.

Fig. 17. Profiles of longitudinal normal stress $\tau_{x x}$ along cylinder wall and wake centerline for UCM fluid at increasing $D e$ (mesh M120).

\section{Acknowledgments}

This work is funded by FEDER funds through the COMPETE 2020 Programme and National Funds through FCT - Portuguese Foundation for Science and Technology under the project UID/CTM/50025/2013 and under the scholarship SFRH/BPD/100353/2014. The author M.S.B. Araujo acknowledges funding from CAPES (Coordenação de Aperfeiçoamento de Pessoal de Nível Superior) proc. BEX 1902-14-8. The authors would like to acknowledge the Minho University cluster under the project Search-ON2: Revitalization of HPC infrastructure of UMinho, (NORTE-07-0162-FEDER-000086), co-funded by the North Portugal Regional Operational Programme (ON.2-0 Novo Norte), under the National Strategic Reference Framework (NSRF), through the European Regional Development Fund (ERDF). The authors thank Professor M.A. Alves and F. Pimenta from University of Porto for insightful comments regarding this work and availability of the deferred correction discretization schemes.

\section{References}

[1] S. Patankar, D. Spalding, Heat and Mass Transfer in Boundary Layers, C.R.C. Press, Cleveland, 1968.

[2] S. Patankar, Numerical Heat Transfer and Fluid Flow, McGraw-Hill, New York, 1980.

[3] J. Ferziger, M. Perić, Computational Methods for Fluid Dynamics, Springer Verlage, Berlin/New York, 2002.

[4] J. Yoo, Y. Na, A numerical study of the planar contraction flow of a viscoelastic fluid using the SIMPLER algorithm, J. Non-Newtonian Fluid Mech. 39 (1991) 89-106.

[5] G. Sasmal, A finite volume approach for calculation of viscoelastic flow through an abrupt axisymmetric contraction, J. Non-Newtonian Fluid Mech. 56 (1995) 15-47.

[6] S.-C. Xue, N. Phan-Thien, R. Tanner, Numerical study of secondary flows of viscoelastic fluid in straight pipes by an implicit finite volume method, J. NonNewtonian Fluid Mech. 59 (1995) 191-213.

[7] X. Huang, N. Phan-Thien, R. Tanner, Viscoelastic flow between eccentric rotating cylinders: unstructured control volume method, J. Non-Newtonian Fluid Mech. 64 (1996) 71-92.

[8] P.J. Oliveira, F.T. Pinho, G. Pinto, Numerical simulation of non-linear elastic flows with a general collocated finite-volume method, J. Non-Newtonian Fluid Mech. 79 (1998) 1-43.

[9] P.J. Oliveira, F.T. Pinho, Plane contraction flows of upper convected maxwell and Phan-Thien-Tanner fluids as predicted by a finite-volume method, J. NonNewtonian Fluid Mech. 88 (1999) 63-88.

[10] M.A. Alves, F.T. Pinho, P.J. Oliveira, Effect of a high-resolution differencing scheme on finite-volume predictions of viscoelastic flows, J. Non-Newtonian Fluid Mech. 93 (2000) 287-314.

[11] M.A. Alves, F.T. Pinho, P.J. Oliveira, The flow of viscoelastic fluids past a cylinder: finite-volume high-resolution methods, J. Non-Newtonian Fluid Mech. 97 (2001) $207-232$.

[12] J. Favero, A. Secchi, N. Cardozo, H. Jasak, Viscoelastic flow analysis using the software openFOAM and differential constitutive equations, J. Non-Newtonian Fluid Mech. 165 (2010) 1625-1636.

[13] F. Habla, A. Obermeier, O. Hinrichsen, Semi-implicit stress formulation for viscoelastic models: application to three-dimensional contraction flows, J. NonNewtonian Fluid Mech. 199 (2013) 70-79.

[14] J. Oldroyd, On the formulation of rheological equations of state, Proc. Roy. Soc. A 200 (1950) 523-541.

[15] J. White, A. Metzner, Development of constitutive equations for polymeric melts and solutions, J. App. Polym. Sci. 7 (1963) 1867-1889.

[16] F. Pimenta, M.A. Alves, Stabilization of an open-source finite volume solver for viscoelastic fluid flows, J. Non-Newtonian Fluid Mech. 239 (2017) 85-104.

[17] R. Owens, T. Phillips, Computational Rheology, Imperial College Press, London, 2002.

[18] R. Guénette, M. Fortin, A new mixed finite element method for computing viscoelastic flows, J. Non-Newtonian Fluid Mech. 60 (1995) 27-52.

[19] P.J. Oliveira, F.T. Pinho, Numerical procedure for the computation of fluid flow 
with arbitrary stress-strain relationships, Numerical Heat Transfer, Part B Fundamentals 35 (1999) 295-315.

[20] F. Habla, A. Woitalka, S. Neuner, O. Hinrichsen, Development of a methodology for numerical simulation of non-isothermal viscoelastic fluid flows with application to axisymmetric 4:1 contraction flows, Chemical Eng. J. 207-208 (2012) 772-784.

[21] X. Chen, H. Marschall, M. Schafer, D. Bothe, A comparison of stabilisation approaches for finite-volume simulation of viscoelastic fluid flow, Int. J. Comp. Fluid Dyn. 27 (2013) 229-250.

[22] F. Habla, M.W. Tan, J. Hablberger, O. Hinrichsen, Numerical simulation of the viscoelastic flow in a three-dimensional lid-driven cavity using the log-conformation reformulation in openFOAM, J. Non-Newtonian Fluid Mech. 212 (2014) 47-62.

[23] C. Fernandes, M.S.B. De Araújo, L.L. Ferrás, J.M. Nóbrega, Improvements in the OpenFOAM numerical code for simulation of steady-state differential viscoelastic flows, Presented by Célio Fernandes at the 11th OpenFOAM Workshop, Guimarães, Portugal, (2016).

[24] S. Patankar, D. Spalding, A calculation procedure for heat, mass and momentum transfer in three-dimensional parabolic flows, Int. Heat Mass Transf. 115 (1972) $1787-1803$.

[25] A. Harten, High resolution schemes for hyperbolic conservation laws, J. Comput. Phys. 49 (1983) 357-393.

[26] P. Gaskell, A. Lau, Curvature-compensated convective transport: SMART, a new boundedness-preserving transport algorithm, Int. J. Numeric. Meth. Fluids 8 (1988) 617-641.

[27] M.A. Alves, P.J. Oliveira, F.T. Pinho, A convergent and universally bounded interpolation scheme for the treatment of advection, Int. J. Numer. Meth. Fluids 41 (2003) 47-75.

[28] H. Jasak, Error Analysis and Estimation for the Finite Volume Method with Applications to Fluid Flows, Ph.D. thesis, Imperial College University of London, 1996.
[29] H. Jasak, H. Weller, Application of the finite volume method and unstructured meshes to linear elasticity, Int. J. Numer. Meth. Engng. 48 (2000) 267-287.

[30] D. Jacobs, Preconditioned Conjugate Gradient Methods for Solving Systems of algebraic equations, Technical Report, RD/L/N193/80, Central Electricity Research Laboratories, 1980.

[31] M. Ajiz, A. Jennings, A robust incomplete Cholesky-conjugate gradient algorithm, J. Numer. Meth. Eng. 20 (1984) 949-966.

[32] J. Lee, J. Zhang, C.-C. Lu, Incomplete LU preconditioning for large scale dense complex linear systems from electromagnetic wave scattering problems, J. NonNewtonian Fluid Mech. 185 (2003) 158-175.

[33] P.J. Oliveira, F.T. Pinho, Analytical solution for fully developed channel and pipe flow of Phan-Thien-Tanner fluids, J. Fluid Mech. 387 (1999) 271-280.

[34] W. Dean, P. Montagnon, On the steady motion of viscous liquid in a corner, Proc. Camb. Philos. Soc. 45 (1949) 389-394.

[35] H. Moffatt, Viscous and resistive eddies near a sharp corner, J. Fluid Mech. 18 (1964) 1-18.

[36] E. Hinch, The flow of an oldroyd fluid around a sharp corner, J. Non-Newtonian Fluid Mech. 50 (1993) 161-171.

[37] M.A. Alves, P.J. Oliveira, F.T. Pinho, Numerical simulations of viscoelastic flow around sharp corners, in: K.J. Bathe (Ed.), Computational Fluid and Solid Mechanics, vol. 2, Elsevier, 2001.

[38] P. Roache, Quantification of uncertainty in computational fluid dynamics, Annu. Rev. Fluid Mech. 29 (1997) 123-160.

[39] R. Comminal, J. Spangenberg, J. Hattel, Robust simulations of viscoelastic flows at high Weissenberg numbers with the streamfunction/log-conformation formulation, J. Non-Newtonian Fluid Mech. 223 (2015) 37-61.

[40] Y. Fan, R. Tanner, N. Phan-Thien, Galerkin/least-square finite-element methods for steady viscoelastic flows, J. Non-Newtonian Fluid Mech. 84 (1999) 233-256. 